Supporting information

(Experimental Procedures)

\title{
Convergent Total Synthesis of (+)-Mycalamide A
}

\author{
Natsuko Kagawa, Masataka Ihara, and Masahiro Toyota* \\ Department of Chemistry, Graduate School of Science, Osaka Prefecture University, Sakai, Osaka \\ 599-8531, Japan \\ E-mail: toyota@c.s.osakafu-u.ac.jp
}

\section{List of Contents}

General

(+)-(2R,3R)-1,2-O-Isopropylidene-4-pentyne-1,2,3-triol (11)

$(2 R S, 3 R, 4 R)$-3-Benzyloxy-4,5-isopropylidenedioxy-1,2-pentanediol (13) S

(+)-(2R,3R,4R)-3-Benzyloxy-1-tert-butyldiphenylsilyloxy-4,5-isopropylidenedioxy-2-pentol (14) $\quad \mathrm{S} 4$

(-)-(2R,3S,4R)-3-Benzyloxy-5-tert-butyldiphenylsilyloxy-1,2,4-pentanetriol. S4

(-)-(2R,3S,4R)-3-Benzyloxy-1-tert-butyldiphenylsilyloxy-2, 4-dihydroxypentan-5-yl pivalate (15) $\quad$ S5

(+)-(2R,3S,4R)-3-Benzyloxy-1-tert-butyldiphenylsilyloxy-2,4-methylenedioxypentanol. S5

(-)-Methyl $(3 S, 4 R, 5 R, 6 R)$-5-Benzyloxy-7-tert-butyldiphenylsilyloxy-2,2-dimethyl-4,6-

methylenedioxy-3-trimethylsilyloxyheptanoate (19a). and (-)-Methyl (3S,4R,5R,6S)-5-Benzyloxy-7- S6

tert-butyldiphenylsilyloxy-2,2-dimethyl-3-hydroxy-4,6-methylenedioxyheptanoate (19b).

(-)-Methyl (3S,4S,5R,6R)-7-tert-Butyldiphenylsilyloxy-2,2-dimethyl-3,5-dihydroxy-4,6-Methylene- $\quad$ S7

dioxyheptanoate.

(+)-Methyl (3S,4S,5R,6R)-7-tert-Butyldiphenylsilyloxy-2,2-dimethyl-3,5-isopropylidenedioxy-4,6-

methylenedioxyheptanoate (19c)

Cinnamaldehyde

(+)-Methyl $(3 S, 4 R, 5 R, 6 R)$-5-Benzyloxy-7-tert-butyldiphenylsilyloxy-2,2-dimethyl-3-methoxy-

4,6-methylenedioxyheptanoate (24)

(+)-(3S,4R,5R,6R)-5-Benzyloxy-7-tert-butyldiphenylsilyloxy-2,2-dimethyl-3-methoxy-4,6-methylene-

dioxyheptanol.

(+)-(3S,4R,5R,6R)-5-Benzyloxy-7-tert-butyldiphenylsilyloxy-2,2-dimethyl-3-methoxy-4,6-methylene-

dioxyheptanal.

(+)-(4S,5R,6R,7R)-6-Benzyloxy-8-tert-butyldiphenylsilyloxy-3,3-dimethyl-4-methoxy-5,7-methylene-

dioxyoct-1-ene $(\mathbf{2 5})$

$(1 R, 5 R, 6 R, 8 R S, 10 S)$-8-Acetoxy-5-(tert-butyldiphenylsilyloxy)-methyl-9,9-dimethyl-10-methoxy-2,4,7- S10

trioxabicyclo[4.4.0]decane $(27)$

(+)-(1R,5R,6R,8R,10S)-5-(tert-Butyl-diphenylsilyloxy)methyl-9,9-dimethyl-10-methoxy-8-(prop-2-

enyl)-2,4,7-trioxabicyclo[4.4.0]decane. and (+)-(1R,5R,6R,8R,10S)-5-(Hydroxy)methyl-10-methoxy-9,9- 
dimethyl-8-(prop-2-enyl)-2,4,7-trioxabicyclo[4.4.0]decane (28)

(+)-(1S,5R,6S,8R,10S)-10-Methoxy-5-\{N-(methoxalyl)- $N$-[(2-trimethylsilyl)ethoxy-carbonyl $]$ amino $\}$ -

9,9-dimethyl-8-(prop-2-enyl)-2,4,7-trioxabicyclo[4.4.0]decane (30)

(+)-(1S,5R,6S,8R,10S)-10-Methoxy-5-\{N-(methoxalyl)amino $\}-9,9-d i m e t h y l-8$-(prop-2-enyl)-2,4,7-

trioxabicyclo[4.4.0]decane (3)

$(2 R, 3 R, 4 R)$-2,3-Epoxy-4,5-isopropylidenedioxypentanol (36)

S13

(-)-(2S,3R,4R)-1-tert-Butyldiphenylsilyloxy-4,5-isopropylidenedioxy-2-mesyloxy-3-methylpentane (41)

S14

(-)-(2R,3R,4R)-4-(4-Methoxybenzyl)oxy-3-methylpentan-1,2-diol (42)

S14

(+)-(3R,4R)-4-(4-Methoxybenzyl)oxy-3-methylpentene (43)

S15

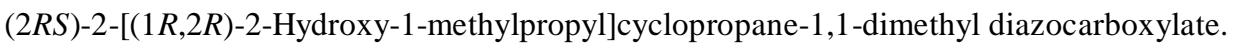

S16

(2R)-2-[(1R,2R)-2-Hydroxy-1-methylpropyl]cyclopropane-1,1-diethyl diazocarboxylate (44). and

(2S)-2-[(1R,2R)-2-Hydroxy-1-methylpropyl]-cyclopropane-1,1-diethyl diazocarboxylate (45).

(4S,5R,6R)-5,6-Dimethyl-4-(Phenylselanylmethyl)tetrahydro-2H-pryan-2-one

(4S,5R,6R)-5,6-Dimethyl-4-(Phenylselanylmethyl)tetrahydro-2H-pryan-2-one

S18

$(2 R, 3 R, 4 R S)$-3,4-Dihydro-2,3-dimethyl-4-phenylselenylmethyl-6-trimethylstannyl-2H-pyran (2) 


\section{Experimental Section}

General. Unless otherwise noted, all reactions were performed in an oven-dried glassware, sealed with a rubber septum under an atmosphere of argon. Anhydrous THF, $\mathrm{CH}_{2} \mathrm{Cl}_{2}$ and $\mathrm{Et}_{2} \mathrm{O}$ were purchased. Anhydrous MeCN, DMF and benzene were purchased. Pyridine, $\mathrm{Et}_{3} \mathrm{~N}, i$ - $\mathrm{Pr}_{2} \mathrm{NEt}, \mathrm{DME}$ and $\mathrm{BF}_{3} \cdot \mathrm{Et}_{2} \mathrm{O}$ were distilled from $\mathrm{CaH}_{2}$ prior to use. Toluene and benzene were distilled from $\mathrm{P}_{2} \mathrm{O}_{5}$. DMSO was distilled from $\mathrm{CaH}_{2}$ under reduced pressure. $\mathrm{Me}_{2} \mathrm{C}(\mathrm{OMe})_{2}$ and $\mathrm{CH}_{2}(\mathrm{OMe})_{2}$ were distilled from $\mathrm{Na}$ and used immediately. $(\mathrm{COCl})_{2}, \mathrm{PivCl}, \mathrm{MsCl}, \mathrm{TiCl}_{4}, \mathrm{TMSCl}, \mathrm{TMSOTf}$, $\mathrm{TMSCH}_{2} \mathrm{CH}_{2} \mathrm{OH}$ and $\mathrm{BnOCH}_{2} \mathrm{C}(\mathrm{O}) \mathrm{Cl}$ were distilled and used immediately. TsCl was dissolved in $\mathrm{Et}_{2} \mathrm{O}$, washed with $10 \% \mathrm{NaOH}$, dried $\left(\mathrm{NaSO}_{4}\right)$ and recrystallized from $\mathrm{Et}_{2} \mathrm{O}$. DMAP was recrystallized from hexane- $\mathrm{CH}_{2} \mathrm{Cl}_{2}-\mathrm{Et}_{2} \mathrm{O}$ and used immediately. Molecular sieves were heated at $130{ }^{\circ} \mathrm{C}$ under reduced pressure for at least 5 hours before use. Unless otherwise noted, materials were obtained from commercial suppliers and used without further purification. Phosphate buffer was prepared by the adjustment of a mixture of $\mathrm{KH}_{2} \mathrm{PO}_{4}\left(1 / 15 \mathrm{M}\right.$ in distilled water) and $\mathrm{Na}_{2} \mathrm{HPO}_{4}$ $(1 / 15 \mathrm{M}$ in distilled water) to $\mathrm{PH}=5.0$ or $\mathrm{PH}=7.0$. Reactions and chromatography fractions were analyzed employing precoated silica gel. Compounds were visualized using an ultraviolet lamp $(254 \mathrm{~nm})$ and/or by staining with $p$-anisaldehyde (in EtOH), phosphomolybdic acid (in EtOH), ammonium molybdate (in $10 \% \quad \mathrm{H}_{2} \mathrm{SO}_{4}$ ). All compounds purified by chromatography are sufficiently pure (>95\% by ${ }^{1} \mathrm{H}$ NMR analysis) for use in subsequent reactions.

(+)-(2R,3R)-1,2- $O$-Isopropylidene-4-pentyne-1,2,3-triol (11). To a $-30{ }^{\circ} \mathrm{C}$ solution of $p$-nitrobenzoic acid $(0.156 \mathrm{~g}, 0.934 \mathrm{mmol})$ and powdered $\mathrm{PPh}_{3}(0.245$

$\mathrm{g}, 0.934 \mathrm{mmol})$ in toluene $(10 \mathrm{~mL})$ was added a solution of anti-alcohol $\mathbf{1 0}(0.178$ $\mathrm{g}, 0.779 \mathrm{mmol})$ in toluene $(3 \mathrm{~mL})$, and followed by a solution of DEAD in toluene (0.424 mL, 40\%, $0.934 \mathrm{mmol})$. The mixture was stirred for $17 \mathrm{~h}$ at $-30{ }^{\circ} \mathrm{C}$, and

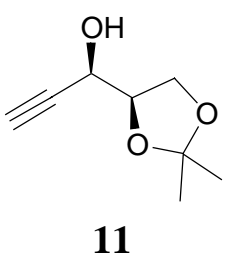
then poured into saturated $\mathrm{NaHCO}_{3}(20 \mathrm{~mL})$. The phases were separated and the aqueous phase was further extracted with $\mathrm{Et}_{2} \mathrm{O}(2 \times 40 \mathrm{~mL})$. The combined organic extracts were washed with brine $(20 \mathrm{~mL})$, dried $\left(\mathrm{MgSO}_{4}\right)$ and concentrated. The resulting yellow solid was diluted with $\mathrm{Et}_{2} \mathrm{O}$ $(9 \mathrm{~mL})$ and hexane $(30 \mathrm{~mL})$, and filtered. The filter cake was washed with $\mathrm{Et}_{2} \mathrm{O}$, and the combined filtrate and washings were evaporated to provide a viscous oil. Purification of the crude product by flash chromatography (9\% EtOAc/hexane) provided syn-p-nitrobenzoate $(0.462 \mathrm{~g},>100 \%)$ as a yellow solid, which was used without further purification in the next step.

To a $0{ }^{\circ} \mathrm{C}$ solution of $s y n$ - $p$-nitrobenzoate $(0.462 \mathrm{~g},<0.779 \mathrm{mmol})$ in $\mathrm{MeOH}(12 \mathrm{~mL})$ was added $\mathrm{K}_{2} \mathrm{CO}_{3}(0.419 \mathrm{~g}, 3.03 \mathrm{mmol})$, and the suspension was stirred for $15 \mathrm{~min}$ at rt. After neutralization with $\mathrm{AcOH}$, the solvent was removed to give a colorless oil. The resulting oil was diluted with $\mathrm{H}_{2} \mathrm{O}(30 \mathrm{~mL})$, and extracted with EtOAc $(2 \times 50 \mathrm{~mL})$. The combined organic extracts were washed with brine $(30 \mathrm{~mL})$, dried $\left(\mathrm{MgSO}_{4}\right)$ and concentrated. Purification of the crude product by flash chromatography (25\% EtOAc/hexane) provided $\beta$-acetylenic alcohol 11 (98.6 mg, 81\% for 2 
steps), of which structure was confirmed by comparison with reported spectral data. ${ }^{1}$

1) Mulzer, J.; Greifenberg, S.; Beckstett, A.; Gottwald, M. Liebigs. Ann. Chem. 1992, 1131.

(2RS,3R,4R)-3-Benzyloxy-4,5-isopropylidenedioxy-1,2-pentanediol (13).

To a solution of olefin $12(2.43 \mathrm{~g}, 9.78 \mathrm{mmol})$ in ${ }^{t} \mathrm{BuOH}-\mathrm{H}_{2} \mathrm{O}(30 \mathrm{~mL}, 1: 1$, $\mathrm{v} / \mathrm{v})$ were added NMO $(1.72 \mathrm{~g}, 14.7 \mathrm{mmol})$ and $\mathrm{OsO}_{4}(0.124 \mathrm{~g}, 0.489 \mathrm{mmol})$.

The mixture was stirred for $10 \mathrm{~h}$ at $\mathrm{rt}$, and then saturated $\mathrm{Na}_{2} \mathrm{SO}_{3}$ was added.

The solution was stirred for an additional $1 \mathrm{~h}$. The resulting mixture was

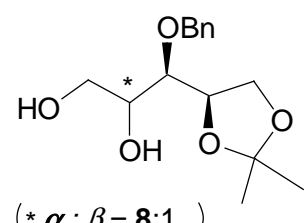

13 extracted with EtOAc (50 mL x 3). The combined organic extracts were washed with brine, dried over $\mathrm{MgSO}_{4}$ and concentrated. Purification of the crude product by flash chromatography (80\% EtOAc/hexane) provided diol $13(2.76 \mathrm{~g}, 100 \%)$ as an 8:1 mixture of diastereoisomers.

Partial data for 13: IR (neat) $3420 \mathrm{~cm}^{-1}$; ${ }^{1} \mathrm{H}$ NMR $\left(300 \mathrm{MHz}, \mathrm{CDCl}_{3}\right) \delta 1.38(\mathrm{~s}, 3 \mathrm{H}), 1.46(\mathrm{~s}, 3 \mathrm{H})$, 2.97 (br s, 2 H), 3.59-3.69 (m, 2 H), 3.71-3.79 (m, 2 H), 3.87 (dd, $J=8.5,7.4$ Hz, 1 H), 4.04 (dd, $J=$ 8.5, 6.6 Hz, $1 \mathrm{H}), 4.32-4.43(\mathrm{~m}, 1 \mathrm{H}), 4.67$ (d, J=11.8 Hz, 0.1 H), 4.68 (d, J=11.5 Hz, 0.9 H), 4.76 $(\mathrm{d}, J=11.3 \mathrm{~Hz}, 0.9 \mathrm{H}), 4.82(\mathrm{~d}, J=11.5 \mathrm{~Hz}, 0.1 \mathrm{H}), 7.27-7.39(\mathrm{~m}, 5 \mathrm{H})$; LRMS $m / z 267\left(\mathrm{M}^{+}-15\right)$; HRMS calcd for $\mathrm{C}_{14} \mathrm{H}_{19} \mathrm{O}_{5} 267.1231$, found: 267.1233 .

(+)-(2R,3R,4R)-3-Benzyloxy-1-tert-butyldiphenylsilyloxy-

4,5-isopropylidenedioxy-2-pentol (14). To a solution of diol $13(0.475$ $\mathrm{g}, 1.68 \mathrm{mmol})$ in $\mathrm{CH}_{2} \mathrm{Cl}_{2}(12 \mathrm{~mL})$ were added $\mathrm{Et}_{3} \mathrm{~N}(0.305 \mathrm{~mL}, 2.18$ mmol), TBDPSCl (0.524 mL, $2.02 \mathrm{mmol})$ and DMAP (10.3 mg, 0.084 mmol). The mixture was stirred for $10 \mathrm{~h}$ at $\mathrm{rt}$, and quenched by $\mathrm{H}_{2} \mathrm{O}$.

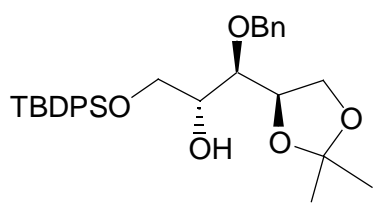

14

The resulting solution was extracted with $\mathrm{Et}_{2} \mathrm{O}(50 \mathrm{~mL} \times 3)$. The combined organic extracts were washed with brine, dried over $\mathrm{MgSO}_{4}$ and concentrated to provide a yellow solid. The crude product was recrystallized from $\mathrm{Et}_{2} \mathrm{O}$-hexane to yield TBDPS ether $14(0.531 \mathrm{~g}, 61 \%)$ as a colorless powder.

Data for 14: $\mathrm{mp} 115-117^{\circ} \mathrm{C} ;[\alpha]^{28}{ }_{\mathrm{D}}=+6.86^{\circ}\left(\mathrm{c} 1.02, \mathrm{CHCl}_{3}\right)$; IR (neat) $3550 \mathrm{~cm}^{-1} ;{ }^{1} \mathrm{H}$ NMR $(300$ $\left.\mathrm{MHz}, \mathrm{CDCl}_{3}\right) \delta 1.07(\mathrm{~s}, 9 \mathrm{H}), 1.36(\mathrm{~s}, 3 \mathrm{H}), 1.43(\mathrm{~s}, 3 \mathrm{H}), 2.71(\mathrm{~d}, J=4.7 \mathrm{~Hz}, 1 \mathrm{H}), 3.51$ (t, $J=6.0$ $\mathrm{Hz}, 1 \mathrm{H}), 3.73-3.85(\mathrm{~m}, 4 \mathrm{H}), 4.01(\mathrm{dd}, J=8.5,6.4 \mathrm{~Hz}, 1 \mathrm{H}), 4.31(\mathrm{dt}, J=7.7,6.2 \mathrm{~Hz}, 1 \mathrm{H}), 4.55$ (d, $J=11.3 \mathrm{~Hz}, 1 \mathrm{H}), 4.74(\mathrm{~d}, J=11.5 \mathrm{~Hz}, 1 \mathrm{H}), 7.19-7.47(\mathrm{~m}, 11 \mathrm{H}), 7.62-7.66(\mathrm{~m}, 4 \mathrm{H}) ;{ }^{13} \mathrm{C}$ NMR $(75$ $\left.\mathrm{MHz}, \mathrm{CDCl}_{3}\right) \delta 138.4,135.8,133.2,133.1,130.1,128.5,128.1,128.0,127.8,109.0,79.2,77.6$, 73.9, 72.0, 66.4, 64.9, 26.9, 26.4, 25.6, 19.2; LRMS $\mathrm{m} / \mathrm{z} 505\left(\mathrm{M}^{+}-15\right)$; HRMS calcd for $\mathrm{C}_{30} \mathrm{H}_{37} \mathrm{O}_{5} \mathrm{Si}$ 505.2408, found: 505.2411; Anal. Calcd for $\mathrm{C}_{31} \mathrm{H}_{40} \mathrm{O}_{5} \mathrm{Si}$ : C, 71.50; H, 7.74. Found: C, $71.34 ; \mathrm{H}, 7.81$.

(-)-(2R,3S,4R)-3-Benzyloxy-5-tert-butyldiphenylsilyloxy1,2,4-pentanetriol. A solution of acetonide $14(6.93 \mathrm{~g}, 13.3 \mathrm{mmol})$ in $\mathrm{AcOH}-\mathrm{THF}-\mathrm{H}_{2} \mathrm{O}(40 \mathrm{~mL}, 3: 1: 1 \mathrm{v} / \mathrm{v})$ was stirred for $6 \mathrm{~h}$ at 55 ${ }^{\circ} \mathrm{C}$. The solution was cooled to $\mathrm{rt}$, and then poured into saturated $\mathrm{NaHCO}_{3}$ solution. The resulting solution was extracted with EtOAc $(100 \mathrm{~mL}$ x 3). The combined organic extracts were washed 
with brine, dried over $\mathrm{MgSO}_{4}$ and concentrated. Purification of the<smiles>O=[R16]([O-])OC[C@@H](O)[C@@H](O)[C@H](O)CO</smiles>
crude product by flash chromatography (80\% EtOAc/hexane) provided a triol $(6.28 \mathrm{~g}, 98 \%)$ as a colorless oil.

Data for triol: $[\alpha]^{26}{ }_{\mathrm{D}}=-5.00^{\circ}\left(\mathrm{c} 1.16, \mathrm{CHCl}_{3}\right)$; IR (neat) $3400 \mathrm{~cm}^{-1} ;{ }^{1} \mathrm{H} \mathrm{NMR}\left(300 \mathrm{MHz}, \mathrm{CDCl}_{3}\right)$ $\delta 1.08$ (s, 9 H), 2.22 (br s, 1 H), 2.92-2.96 (m, 2 H), 3.59 (dd, J= 3.2, 6.4 Hz, 1 H), 3.63-3.73 (m, 2 H), 3.77 (dd, $J=6.1,10.4 \mathrm{~Hz}, 1 \mathrm{H}), 3.85(\mathrm{dd}, J=4.4,10.4 \mathrm{~Hz}, 1 \mathrm{H}), 3.88-3.97$ (m, $2 \mathrm{H}), 4.51$ (d, $J=$ $11.3 \mathrm{~Hz}, 1 \mathrm{H}), 4.56(\mathrm{~d}, J=11.3 \mathrm{~Hz}, 1 \mathrm{H}), 7.16-7.21(\mathrm{~m}, 2 \mathrm{H}), 7.26-7.30(\mathrm{~m}, 3 \mathrm{H}), 7.35-7.48(\mathrm{~m}, 6 \mathrm{H})$, 7.63-7.66 (m, $4 \mathrm{H}) ;{ }^{13} \mathrm{C} \mathrm{NMR}\left(75 \mathrm{MHz}, \mathrm{CDCl}_{3}\right) \delta 137.6,135.7,135.7,133.1,133.0,130.0,128.6$, 128.2, 128.1, 128.0, 78.1, 73.4, 71.6, 64.8, 64.0, 26.9, 19.1; LRMS $m / z 423$ (M+57); HRMS calcd for $\mathrm{C}_{24} \mathrm{H}_{27} \mathrm{O}_{5} \mathrm{Si}$ 423.1626, found: 423.1631; Anal. Calcd for $\mathrm{C}_{28} \mathrm{H}_{36} \mathrm{O}_{5} \mathrm{Si}$ : C, 69.96; H, 7.54. Found: C, 69.99; H, 7.63 .

\section{(-)-(2R,3S,4R)-3-Benzyloxy-1-tert-butyldiphenylsilyloxy-}

2, 4-dihydroxypentan-5-yl pivalate (15). To a solution of the above triol $(1.21 \mathrm{~g}, 2.51 \mathrm{mmol})$ in $\mathrm{CH}_{2} \mathrm{Cl}_{2}(10 \mathrm{~mL})$ were added pyridine $(2 \mathrm{~mL}, 24.7 \mathrm{mmol})$ and $\mathrm{PivCl}(0.324 \mathrm{~mL}, 2.63 \mathrm{mmol})$. The mixture was stirred for $10 \mathrm{~h}$ at $\mathrm{rt}$. After the solvent was removed,

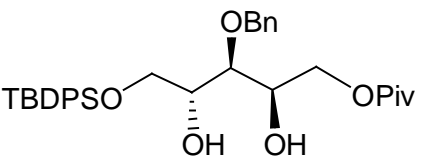

15 the resulting crude oil was purified by flash chromatography (20\% EtOAc/hexane) to provide pivalate $15(1.27 \mathrm{~g}, 89 \%)$ as a colorless oil.

Data for 15: $[\alpha]^{20}{ }_{\mathrm{D}}=-4.50^{\circ}$ (c 1.20, $\left.\mathrm{CHCl}_{3}\right)$; IR (neat) $3450,1725 \mathrm{~cm}^{-1} ;{ }^{1} \mathrm{H} \mathrm{NMR}(300 \mathrm{MHz}$, $\left.\mathrm{CDCl}_{3}\right) \delta 1.08(\mathrm{~s}, 9 \mathrm{H}), 1.21(\mathrm{~s}, 9 \mathrm{H}), 2.79(\mathrm{br} \mathrm{s}, 2 \mathrm{H}), 3.58(\mathrm{dd}, J=1.9,7.1 \mathrm{~Hz}, 1 \mathrm{H}), 3.78(\mathrm{dd}, J=$ 5.5, $10.4 \mathrm{~Hz}, 1 \mathrm{H}), 3.87$ (dd, J=3.8, $10.4 \mathrm{~Hz}, 1 \mathrm{H}), 3.90-3.97(\mathrm{~m}, 1 \mathrm{H}), 4.12(\mathrm{dd}, J=5.5,12.7 \mathrm{~Hz}, 1$ H), 4.11-4.15 (m, $1 \mathrm{H}), 4.25$ (dd, $J=8.8,12.6 \mathrm{~Hz}, 1 \mathrm{H}), 4.53$ (s, $2 \mathrm{H}), 7.14-7.18$ (m, $2 \mathrm{H}), 7.26-7.29$ $(\mathrm{m}, 3 \mathrm{H}), 7.32-7.48(\mathrm{~m}, 6 \mathrm{H}), 7.63-7.66(\mathrm{~m}, 4 \mathrm{H}) ;{ }^{13} \mathrm{C} \mathrm{NMR}\left(75 \mathrm{MHz}, \mathrm{CDCl}_{3}\right) \delta 178.5,137.5$, 135.6, 135.6, 133.0, 132.8, 130.0, 128.5, 128.1, 128.0, 127.9, 77.2, 73.6, 71.4, 69.1, 64.9, 64.7, 38.6, 27.1, 26.8, 19.1; LRMS m/z 489 ( $\left.\mathrm{M}^{+}-75\right)$; HRMS calcd for $\mathrm{C}_{29} \mathrm{H}_{33} \mathrm{O}_{5} \mathrm{Si}$ 489.2095, found: 489.2099; Anal. Calcd for $\mathrm{C}_{33} \mathrm{H}_{44} \mathrm{O}_{6} \mathrm{Si}$ : C, 70.17; H, 7.85. Found: C, 70.00; H, 7.81.

\section{(+)-(2R,3S,4R)-3-Benzyloxy-1-tert-butyldiphenylsilyloxy-}

2,4-methylenedioxypentanol. To a $0{ }^{\circ} \mathrm{C}$ solution of $\mathbf{1 6}(1.22 \mathrm{~g}, 2.12$

$\mathrm{mmol})$ in THF $(13 \mathrm{~mL})$ was added dropwise DIBAL-H $(4.96 \mathrm{~mL}$, $4.66 \mathrm{mmol})$. The solution was stirred for $1 \mathrm{~h}$ at the same temperature.

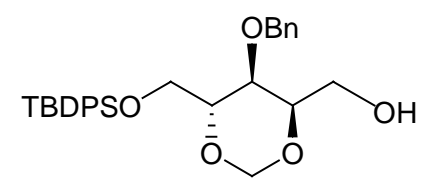
$\mathrm{H}_{2} \mathrm{O}(5 \mathrm{~mL})$ was added to quench the reaction. After $20 \mathrm{~min}, \mathrm{Et}_{2} \mathrm{O}$ $(10 \mathrm{~mL})$ and hexane $(10 \mathrm{~mL})$ were added. The resulting mixture was allowed to warm to $\mathrm{rt}$ and stirred for $10 \mathrm{~h}$, and then dried $\left(\mathrm{MgSO}_{4}\right)$ and filterd through Celite. The filtrate was concentrated to afford a crude oil. Purification of the crude product by flash chromatography (33\% EtOAc/hexane) provided the corresponding alcohol (1.04 g, 99\%) as a colorless oil.

Data for alcohol: $[\alpha]^{27}{ }_{\mathrm{D}}=+0.75^{\circ}\left(\mathrm{c} 1.05, \mathrm{CHCl}_{3}\right)$; IR (neat) $3425 \mathrm{~cm}^{-1}$; ${ }^{1} \mathrm{H}$ NMR $(300 \mathrm{MHz}$, $\left.\mathrm{CDCl}_{3}\right) \delta 1.08(\mathrm{~s}, 9 \mathrm{H}), 1.83-1.85(\mathrm{~m}, 1 \mathrm{H}), 3.62-3.71(\mathrm{~m}, 2 \mathrm{H}), 3.89-3.96(\mathrm{~m}, 4 \mathrm{H}), 3.98-4.01$ (m, 
$1 \mathrm{H}), 4.46(\mathrm{~d}, J=11.5 \mathrm{~Hz}, 1 \mathrm{H}), 4.67(\mathrm{~d}, J=11.8 \mathrm{~Hz}, 1 \mathrm{H}), 4.83(\mathrm{~d}, J=6.3 \mathrm{~Hz}, 1 \mathrm{H}), 4.91(\mathrm{~d}, J=6.3$ $\mathrm{Hz}, 1 \mathrm{H}), 7.25-7.48(\mathrm{~m}, 11 \mathrm{H}), 7.64-7.68(\mathrm{~m}, 4 \mathrm{H}) ;{ }^{13} \mathrm{C}$ NMR $\left(75 \mathrm{MHz}, \mathrm{CDCl}_{3}\right) \delta 137.5,135.8$, 135.7, 133.1, 130.0, 128.7, 128.3, 128.2, 127.9, 127.9, 88.2, 75.0, 74.4, 72.0, 70.8, 62.2, 61.0, 26.8, 19.2; LRMS $m / z 435\left(\mathrm{M}^{+}-57\right)$; HRMS calcd for $\mathrm{C}_{25} \mathrm{H}_{27} \mathrm{O}_{5} \mathrm{Si}$ 435.1626, found: 435.1622; Anal. Calcd for $\mathrm{C}_{29} \mathrm{H}_{36} \mathrm{O}_{5} \mathrm{Si}$ : C, 70.69; H, 7.36. Found: C, 70.83; H, 7.51.

\section{(-)-Methyl (3S,4R,5R,6R)-5-Benzyloxy-7-tert-butyldiphenylsilyloxy-2,2-dimethyl-4,6-} methylenedioxy-3-trimethylsilyloxyheptanoate (19a). (-)-Methyl (3S,4R,5R,6S)-5-Benzyloxy-7-tert-butyldiphenylsilyloxy-2,2-dimethyl-3hydroxy-4,6-methylenedioxyheptanoate (19b).

(entry 1) To a water bath cooled suspention of $\mathrm{Yb}(\mathrm{OTf})_{3}(47$ $\mathrm{mg}, 0.076 \mathrm{mmol})$ in $\mathrm{CH}_{2} \mathrm{Cl}_{2}(30 \mathrm{~mL})$ was added dropwise a mixture of aldehyde $17(0.38 \mathrm{~g})$ and methyl trimethylsilyl dimethylketene acetal $(0.39 \mathrm{~mL}, 1.92 \mathrm{mmol})$ in $\mathrm{CH}_{2} \mathrm{Cl}_{2}(10$

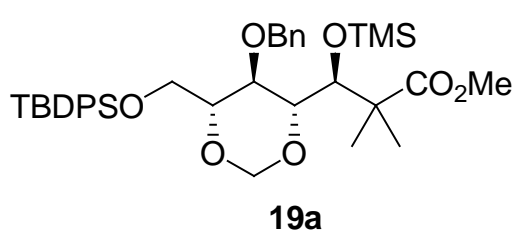
$\mathrm{mL}$ ). The mixture was stirred for $48 \mathrm{~h}$ at $\mathrm{rt}$ and saturated $\mathrm{NaHCO}_{3}$ solution $(10 \mathrm{~mL})$ was added. The phases were separated and the aqueous phase was further extracted with EtOAc (50 mL x 3). The combined organic extracts were washed

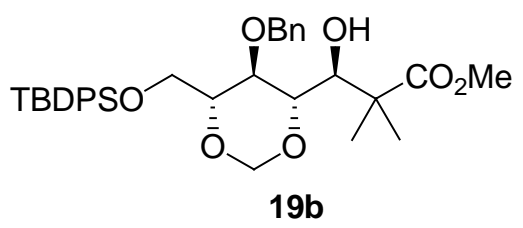
with brine, dried over $\mathrm{MgSO}_{4}$ and concentrated. The crude product was purified by flash chromatography. Elution with a 10:1 mixture of hexane-EtOAc afforded ethers 18a and 19a (18a: $0.261 \mathrm{~g}, 51 \%$ for 2 steps; 19a: $0.125 \mathrm{~g}, 25 \%$ for 2 steps) as a colorless oil. Elution with a 6:1 mixture of hexane-EtOAc afforded aldols $\mathbf{1 8 b}$ and 19b (18b: 35.7 $\mathrm{mg}, 8 \%$ for 2 steps; 19b: $24.0 \mathrm{mg}$, 5\% for 2 steps) as a colorless oil.

Data for 19a: $[\alpha]_{D}^{27}=-1.64^{\circ}\left(\mathrm{c} 1.68, \mathrm{CHCl}_{3}\right)$; IR (neat) $2932,1732,1252,1113,1042,843,735$, $702 \mathrm{~cm}^{-1}$; ${ }^{1} \mathrm{H}$ NMR $\left(500 \mathrm{MHz}, \mathrm{CDCl}_{3}\right) \delta 0.21(\mathrm{~s}, 9 \mathrm{H}), 1.07(\mathrm{~s}, 9 \mathrm{H}), 1.20(\mathrm{~s}, 3 \mathrm{H}), 1.28(\mathrm{~s}, 3 \mathrm{H})$, $3.41(\mathrm{td}, J=2.4,7.2 \mathrm{~Hz}, 1 \mathrm{H}), 3.45(\mathrm{~d}, J=7.6 \mathrm{~Hz}, 1 \mathrm{H}), 3.62(\mathrm{~s}, 3 \mathrm{H}), 3.82(\mathrm{t}, J=7.2 \mathrm{~Hz}, 1 \mathrm{H})$, 3.92-3.93 (m, 2 H), 4.42 (s, $1 \mathrm{H}), 4.59$ (d, $J=4.8 \mathrm{~Hz}, 1 \mathrm{H}), 4.72$ (d, $J=3.6 \mathrm{~Hz}, 1 \mathrm{H}), 5.15$ (d, $J=4.8$ $\mathrm{Hz}, 1 \mathrm{H}), 7.19-7.21$ (m, $2 \mathrm{H}), 7.27-7.41(\mathrm{~m}, 9 \mathrm{H}), 7.67-7.70(\mathrm{~m}, 2 \mathrm{H}), 7.72-7.74(\mathrm{~m}, 2 \mathrm{H}) ;{ }^{13} \mathrm{C} \mathrm{NMR}$ $\left(125 \mathrm{MHz}, \mathrm{CDCl}_{3}\right) \delta 177.3,137.9,135.9,135.7,133.5,132.9,129.7,129.6,128.4,127.7,127.6$, 127.3, 92.8, 81.8, 79.2, 74.0, 73.5, 69.8, 63.4, 51.8, 48.2, 26.9, 21.9, 19.3, 1.2; LRMS m/z 607 $\left(\mathrm{M}^{+}-57\right)$; HRMS calcd for $\mathrm{C}_{33} \mathrm{H}_{43} \mathrm{O}_{7} \mathrm{Si}_{2}$ 607.2545, found: 607.2552.

Data for 19b: $[\alpha]^{28}=-3.09^{\circ}\left(\mathrm{c} 0.34, \mathrm{CHCl}_{3}\right)$; IR (neat) 3452, 1732, 1084, 1040, 741, $702 \mathrm{~cm}^{-1}$; ${ }^{1} \mathrm{H}$ NMR $\left(300 \mathrm{MHz}, \mathrm{CDCl}_{3}\right) \delta 1.07$ (s, $\left.9 \mathrm{H}\right), 1.20(\mathrm{~s}, 3 \mathrm{H}), 1.34(\mathrm{~s}, 3 \mathrm{H}), 3.42(\mathrm{~d}, J=10.9 \mathrm{~Hz}, 1 \mathrm{H})$, $3.74(\mathrm{~d}, J=10.9 \mathrm{~Hz}, 1 \mathrm{H}), 3.48(\mathrm{td}, J=2.4,9.0 \mathrm{~Hz}, 1 \mathrm{H}), 3.52(\mathrm{~d}, J=9.3 \mathrm{~Hz}, 1 \mathrm{H}), 3.67$ (s, $3 \mathrm{H})$, 3.89-3.97 (m, $3 \mathrm{H}), 4.59$ (d, $J=5.4 \mathrm{~Hz}, 1 \mathrm{H}), 4.61$ (d, $J=10.2 \mathrm{~Hz}, 1 \mathrm{H}), 4.70(\mathrm{~d}, J=10.2 \mathrm{~Hz}, 1 \mathrm{H})$, 4.99 (d, J=5.8 Hz, $1 \mathrm{H}), 7.16-7.19$ (m, $2 \mathrm{H}), 7.27-7.45$ (m, $9 \mathrm{H}), 7.64-7.73$ (m, $4 \mathrm{H}) ;{ }^{13} \mathrm{C}$ NMR $(75$ $\left.\mathrm{MHz}, \mathrm{CDCl}_{3}\right) \delta 177.7,138.0,135.7,133.3,129.7,129.7,128.5,127.9,127.8,127.6,92.6, \quad 81.5$, 78.8, 74.9, 74.7, 69.4, 65.8, 63.2, 51.8, 44.5, 26.7, 24.3, 21.7, 19.2, 15.1; LRMS m/z $535\left(\mathrm{M}^{+}-57\right)$; HRMS calcd for $\mathrm{C}_{30} \mathrm{H}_{35} \mathrm{O}_{7} \mathrm{Si} 535.2150$, found: 535.2154. 
(entry 2) To a $-78{ }^{\circ} \mathrm{C}$ solution of aldehyde $17(53.0 \mathrm{mg})$ in $\mathrm{CH}_{2} \mathrm{Cl}_{2}(1 \mathrm{~mL})$ was added dropwise $\mathrm{TiCl}_{4}(0.0180 \mathrm{~mL}, 0.162 \mathrm{mmol})$, followed by methyl trimethylsilyl dimethylketene acetal $(0.0330$ $\mathrm{mL}, 0.162 \mathrm{mmol})$ in $\mathrm{CH}_{2} \mathrm{Cl}_{2}(1 \mathrm{~mL})$. The mixture was stirred for $1 \mathrm{~h}$ at $-78{ }^{\circ} \mathrm{C}$ and quenched by $\mathrm{H}_{2} \mathrm{O}$. The phases were separated and the aqueous phase was further extracted with $\mathrm{Et}_{2} \mathrm{O}(10 \mathrm{~mL} x$ 3). The combined organic extracts were washed with brine, dried over $\mathrm{MgSO}_{4}$ and concentrated. Purification of the crude product by flash chromatography (20\% EtOAc/hexane) provided aldol 18b (40.4 mg, 63\% for 2 steps).

\section{(-)-Methyl (3S,4S,5R,6R)-7-tert-Butyldiphenylsilyloxy-2,2-dimethyl-3,5-dihydroxy-4,6-}

methylenedioxyheptanoate. To a solution of benzyl ether $19 \mathrm{~b}(53.7 \mathrm{mg}, 0.0906 \mathrm{mmol})$ in EtOH $(1.5 \mathrm{~mL})$ was added 10\% Pd-C (3.0 mg). Hydrogen was flushed through the flask and the solution was stirred under $\mathrm{H}_{2}$ for 1 week. The system was flushed with $\mathrm{Ar}(\mathrm{g})$ and then filtered through Celite. The filtrate was concentrated to afford a crude oil. Purification of the crude product by flash chromatography (20\% EtOAc/hexane) provided the corresponding diol $(25.0 \mathrm{mg}, 55 \%)$ as a colorless oil, in addition to recovered benzyl ether $19 \mathrm{~b}(9.5 \mathrm{mg}, 18 \%)$.

Data for diol: $[\alpha]^{28}{ }_{\mathrm{D}}=-14.69^{\circ}\left(\mathrm{c} 0.48, \mathrm{CHCl}_{3}\right.$ ); IR (neat) $3447,1717,1084,704 \mathrm{~cm}^{-1} ;{ }^{1} \mathrm{H} \mathrm{NMR}$ $\left(300 \mathrm{MHz}, \mathrm{CDCl}_{3}\right) \delta 1.06(\mathrm{~s}, 9 \mathrm{H}), 1.23(\mathrm{~s}, 3 \mathrm{H}), 1.34(\mathrm{~s}, 3 \mathrm{H}), 3.08(\mathrm{~d}, J=2.4 \mathrm{~Hz}, 1 \mathrm{H}), 3.28(\mathrm{~d}, J=$ $10.4 \mathrm{~Hz}, 1 \mathrm{H}), 3.48-3.57$ (m, $2 \mathrm{H}), 3.66(\mathrm{~s}, 3 \mathrm{H}), 3.78-3.98(\mathrm{~m} 4 \mathrm{H}), 4.62(\mathrm{~d}, J=5.8 \mathrm{~Hz}, 1 \mathrm{H}), 4.92$ $(\mathrm{d}, J=5.8 \mathrm{~Hz}, 1 \mathrm{H}), 7.38-7.49$ (m, $6 \mathrm{H}), 7.66(\mathrm{dd}, J=2.0,2.0 \mathrm{~Hz}, 2 \mathrm{H}), 7.69$ (dd, $J=1.3,3.0 \mathrm{~Hz}, 2$ $\mathrm{H}) ;{ }^{13} \mathrm{C}$ NMR $\left(75 \mathrm{MHz}, \mathrm{CDCl}_{3}\right) \delta 177.6,135.6,132.5,130.1,127.9,92.5,78.9,78.6,74.0,65.9$, 65.2, 51.8, 44.5, 26.7, 21.6, -18.9; LRMS $m / z 501\left(\mathrm{M}^{+}-1\right)$; HRMS calcd for $\mathrm{C}_{27} \mathrm{H}_{37} \mathrm{O}_{7} \mathrm{Si} 501.2309$, found: 501.2312; Anal. Calcd for $\mathrm{C}_{27} \mathrm{H}_{38} \mathrm{O}_{7} \mathrm{Si}$ : C, 64.51; H, 7.62. Found: C, 64.14; H, 7.26.

\section{(+)-Methyl (3S,4S,5R,6R)-7-tert-Butyldiphenylsilyloxy-2,2-dimethyl-3,5-isopropylidene-}

dioxy-4,6-methylenedioxyheptanoate (19c). To a solution of the above diol (11.4 mg, $0.0227 \mathrm{mmol})$ in DMF $(1 \mathrm{~mL})$ were added dimethoxypropane $(3 \mathrm{~mL})$ and $\mathrm{TsOH} \cdot \mathrm{H}_{2} \mathrm{O}(1.0 \mathrm{mg}$, cat.). The mixture was stirred for 1 week, and then poured into saturated $\mathrm{NaHCO}_{3}$ solution $(3 \mathrm{~mL})$. The phases were

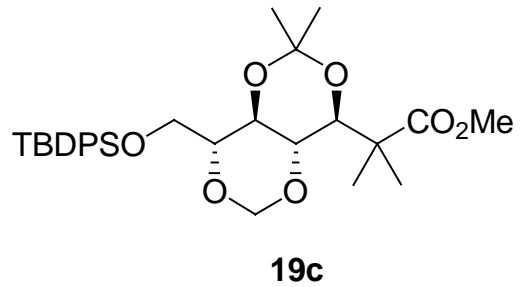
separated and the aqueous phase was extracted with EtOAc $(15 \mathrm{~mL} \times 3)$. The combined organic extracts were washed with brine, dried over $\mathrm{MgSO}_{4}$ and concentrated. Purification of the crude product by flash chromatography (17\% EtOAc/hexane) provided acetonide 19c $(3.8 \mathrm{mg}, 31 \%)$ as a colorless oil, in addition to recovered the starting material $(4.1 \mathrm{mg}, 36 \%)$.

Data for 19c: $[\alpha]^{27}=+3.82^{\circ}\left(\mathrm{c} 0.38, \mathrm{CHCl}_{3}\right)$; IR (neat) 1730, 1227, 1016, $702 \mathrm{~cm}^{-1} ;{ }^{1} \mathrm{H}$ NMR $\left(300 \mathrm{MHz}, \mathrm{CDCl}_{3}\right) \delta 1.05(\mathrm{~s}, 9 \mathrm{H}), 1.27(\mathrm{~s}, 3 \mathrm{H}), 1.34(\mathrm{~s}, 3 \mathrm{H}), 1.36(\mathrm{~s}, 3 \mathrm{H}), 1.38(\mathrm{~s}, 3 \mathrm{H}), 3.48(\mathrm{td}$, $J=3.0,8.6 \mathrm{~Hz}, 1 \mathrm{H}), 3.66(\mathrm{~s}, 3 \mathrm{H}), 3.73(\mathrm{dd}, J=7.6,9.4 \mathrm{~Hz}, 1 \mathrm{H}) 3.81-3.87$ (m $3 \mathrm{H}), 4.45(\mathrm{~d}, J=7.7$ $\mathrm{Hz}, 1 \mathrm{H}), 4.58(\mathrm{~d}, J=6.0 \mathrm{~Hz}, 1 \mathrm{H}), 5.05(\mathrm{~d}, J=6.0 \mathrm{~Hz}, 1 \mathrm{H}), 7.34-7.45(\mathrm{~m}, 6 \mathrm{H}), 7.69-7.73(\mathrm{~m}, 4 \mathrm{H})$; ${ }^{13} \mathrm{C}$ NMR $\left(75 \mathrm{MHz}, \mathrm{CDCl}_{3}\right) \delta 177.4,135.9,135.7,129.6,127.6,101.2,93.6,80.8,78.6,72.3,62.7$, 62.6, 51.6, 44.6, 27.1, 26.6, 24.1, 19.2, 18.4; LRMS $\mathrm{m} / \mathrm{z} 527\left(\mathrm{M}^{+}-15\right)$; HRMS calcd for 
$\mathrm{C}_{29} \mathrm{H}_{39} \mathrm{O}_{7} \mathrm{Si}$ 527.2465, found: 527.2470.

Cinnamaldehyde. To a suspension of $\mathrm{Yb}(\mathrm{OTf})_{3}(305 \mathrm{mg}, 0.492 \mathrm{mmol})$ and TMSCl (1.37 mL, $10.8 \mathrm{mmol})$ in $\mathrm{CH}_{2} \mathrm{Cl}_{2}(5 \mathrm{~mL})$ were added benzaldehyde $(0.05 \mathrm{~mL}, 0.492 \mathrm{mmol})$ and $\mathrm{Et}_{3} \mathrm{~N}(3.43 \mathrm{~mL}, 24.6 \mathrm{mmol})$ at $\mathrm{rt}$, and then the<smiles>O=C/C=C/c1ccccc1</smiles>
resulting mixture was stirred at $30{ }^{\circ} \mathrm{C}$ for $24 \mathrm{~h}$. Saturated $\mathrm{NaHCO}_{3}$ solution was added at $15{ }^{\circ} \mathrm{C}$. After separation, the aqueous layer was extracted twice with EtOAc. The combined organic layers were washed with brine, dried $\left(\mathrm{MgSO}_{4}\right)$ and concentrated. Purification of the crude product by flash chromatography (10\% EtOAc/hexane) yielded cinnamaldehyde (22.9 mg, 35\%) as an oil.

\section{(+)-Methyl (3S,4R,5R,6R)-5-Benzyloxy-7-tert-butyldiphenylsilyloxy-2,2-dimethyl-}

3-methoxy-4,6-methylenedioxy-heptanoate (24). To a solution of a crude mixture of $\mathbf{1 8 a}$ and $\mathbf{1 8 b}(16 \mathrm{mg})$ in $\mathrm{MeOH}(1 \mathrm{~mL})$ was added CSA (1.0 mg, cat.). The mixture was stirred for $15 \mathrm{~min}$ at $\mathrm{rt}$, thenquenched by saturated

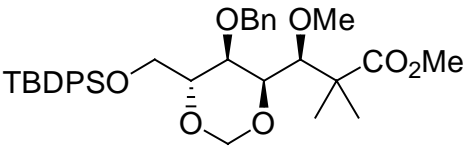

24 $\mathrm{NaHCO}_{3}(1 \mathrm{~mL})$. The phases were separated and the aqueous phase was extracted with EtOAc. The combined organic extracts were washed with brine, dried over $\mathrm{MgSO}_{4}$ and concentrated. Purification of the crude product by flash chromatography (14\% EtOAc/hexane) provided alcohol $\mathbf{1 8 b}(11.9 \mathrm{mg}, 84 \%$ for $3 \mathrm{steps})$.

To a $0{ }^{\circ} \mathrm{C}$ suspension of $\mathrm{NaH}(53.7 \mathrm{mg}, 1.34 \mathrm{mmol})$ in THF $(13 \mathrm{~mL})$ was added dropwise a solution of alcohol $\mathbf{1 8 b}(0.531 \mathrm{~g}, 0.895 \mathrm{mmol})$ in THF $(2 \mathrm{~mL})$. The mixture was stirred for $30 \mathrm{~min}$ at $0{ }^{\circ} \mathrm{C}$, then warmed to $\mathrm{rt}$ and stirred for $1 \mathrm{~h}$. After the solution was cooled to $0{ }^{\circ} \mathrm{C}$, MeI $(0.111 \mathrm{~mL}$, $1.79 \mathrm{mmol}$ ) was added and the mixture was stirred for $30 \mathrm{~min}$ at $0{ }^{\circ} \mathrm{C}$. The resulting mixture was allowed to warm to $\mathrm{rt}$ and stirred for an additional $4 \mathrm{~h}$, then poured into saturated $\mathrm{NH}_{4} \mathrm{Cl}$ solution at $0{ }^{\circ} \mathrm{C}$. The phases were separated and the aqueous phase was extracted with $\mathrm{Et}_{2} \mathrm{O}$. The combined organic extracts were washed with brine, dried over $\mathrm{MgSO}_{4}$ and concentrated. Purification of the crude product by flash chromatography (13\% EtOAc/hexane) provided methyl ether $24(0.519 \mathrm{mg}$, $95 \%)$.

Data for 24: $[\alpha]^{20}{ }_{\mathrm{D}}=+28.18^{\circ}\left(\mathrm{c} 0.85, \mathrm{CHCl}_{3}\right.$ ); IR (neat) $1720 \mathrm{~cm}^{\square 1} ;{ }^{1} \mathrm{H} \mathrm{NMR}\left(300 \mathrm{MHz}, \mathrm{CDCl}_{3}\right)$ $\delta 1.08(\mathrm{~s}, 9 \mathrm{H}), 1.17$ (s, $3 \mathrm{H}), 1.26(\mathrm{~s}, 3 \mathrm{H}), 3.38$ (s, $3 \mathrm{H}), 3.65(\mathrm{~s}, 3 \mathrm{H}), 3.83(\mathrm{~d}, J=2.7 \mathrm{~Hz}, 1 \mathrm{H})$, 3.86-3.93 (m, $3 \mathrm{H}), 4.06-4.13(\mathrm{~m}, 2 \mathrm{H}), 4.55(\mathrm{~d}, J=11.5 \mathrm{~Hz}, 1 \mathrm{H}), 4.62(\mathrm{~d}, J=11.8 \mathrm{~Hz}, 1 \mathrm{H}), 4.74(\mathrm{~d}$, $J=5.5 \mathrm{~Hz}, 1 \mathrm{H}), 5.26(\mathrm{~d}, J=5.5 \mathrm{~Hz}, 1 \mathrm{H}), 7.26-7.42(\mathrm{~m}, 11 \mathrm{H}), 7.67-7.73(\mathrm{~m}, 4 \mathrm{H}) ;{ }^{13} \mathrm{C} \mathrm{NMR}(75$ $\left.\mathrm{MHz}, \mathrm{CDCl}_{3}\right) \delta 177.6,138.1,135.9,135.8,133.7,133.4,129.8,128.6,128.1,128.0,127.8,127.8$, 90.0, 84.9, 75.4, 72.7, 72.6, 71.6, 63.3, 60.5, 51.8, 47.3, 26.8, 21.9, 21.8, 19.3; LRMS m/z 457 $\left(\mathrm{M}^{+}-149\right)$; HRMS calcd for $\mathrm{C}_{24} \mathrm{H}_{29} \mathrm{O}_{7} \mathrm{Si}$ 457.1681, found: 457.1679; Anal. Calcd for $\mathrm{C}_{35} \mathrm{H}_{46} \mathrm{O}_{7} \mathrm{Si}$ : C,69.27; H, 7.64. Found: C, 69.27; H, 7.77.

(+)-(3S,4R,5R,6R)-5-Benzyloxy-7-tert-butyldiphenylsilyloxy -2,2-dimethyl-3-methoxy-4,6-methylenedioxyheptanol. To a

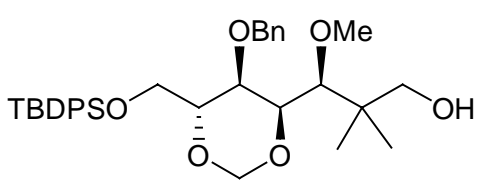


$0{ }^{\circ} \mathrm{C}$ solution of ester $24(0.0980 \mathrm{~g}, 0.162 \mathrm{mmol})$ in THF $(5 \mathrm{~mL})$ was added dropwise DIBAL-H $(0.440 \mathrm{~mL}, 0.404 \mathrm{mmol})$. The solution was stirred for $1 \mathrm{~h}$ at the same temperature. $\mathrm{H}_{2} \mathrm{O}(0.44 \mathrm{~mL})$ was added to quench the reaction. After $20 \mathrm{~min}, \mathrm{Et}_{2} \mathrm{O}(5 \mathrm{~mL})$ and hexane $(5 \mathrm{~mL})$ were added. The resulting mixture was allowed to warm to rt and stirred for $1 \mathrm{~h}$, and then dried $\left(\mathrm{MgSO}_{4}\right)$ and filterd through Celite. The filtrate was concentrated to afford a crude solid. The crude product was recrystallized from $\mathrm{Et}_{2} \mathrm{O}$-hexane to provide the corresponding alcohol $(0.0934 \mathrm{~g}, 100 \%)$ as a colorless plate.

Data for alcohol: $\mathrm{mp} 108-109{ }^{\circ} \mathrm{C} ;[\alpha]^{27}{ }_{\mathrm{D}}=+30.36^{\circ}$ (c 1.05, $\mathrm{CHCl}_{3}$ ); IR (neat) $3450 \mathrm{~cm}^{-1} ;{ }^{1} \mathrm{H}$ NMR (300 MHz, $\left.\mathrm{CDCl}_{3}\right) \delta 0.86(\mathrm{~s}, 3 \mathrm{H}), 0.94(\mathrm{~s}, 3 \mathrm{H}), 1.09(\mathrm{~s}, 9 \mathrm{H}), 2.99(\mathrm{dd}, J=5.5,6.9 \mathrm{~Hz}, 1 \mathrm{H})$, 3.37 (d, $J=2.5 \mathrm{~Hz}, 1 \mathrm{H}), 3.43$ (s, $3 \mathrm{H}), 3.44-3.54$ (m, $2 \mathrm{H}), 3.92-3.96$ (m, $3 \mathrm{H}), 4.06-4.12(\mathrm{~m}, 1 \mathrm{H})$, $4.15(\mathrm{dd}, J=2.2,5.8 \mathrm{~Hz}, 1 \mathrm{H}), 4.52(\mathrm{~d}, J=11.5 \mathrm{~Hz}, 1 \mathrm{H}), 4.65(\mathrm{~d}, J=11.5 \mathrm{~Hz}, 1 \mathrm{H}), 4.81$ (d, $J=5.5$ $\mathrm{Hz}, 1 \mathrm{H}), 5.36(\mathrm{~d}, J=5.5 \mathrm{~Hz}, 1 \mathrm{H}), 7.25-7.45(\mathrm{~m}, 11 \mathrm{H}), 7.68-7.74(\mathrm{~m}, 4 \mathrm{H}) ;{ }^{13} \mathrm{C} \mathrm{NMR}(75 \mathrm{MHz}$, $\left.\mathrm{CDCl}_{3}\right) \delta 138.0,136.0,135.8,133.7,133.4,129.9,128.7,128.1,127.9,127.8,90.3,87.0,75.7$, 72.9, 72.1, 71.6, 69.2, 63.4, 60.9, 40.5, 26.9, 24.0, 21.6, 19.3; LRMS m/z $521\left(\mathrm{M}^{+}-57\right)$; HRMS calcd for $\mathrm{C}_{30} \mathrm{H}_{37} \mathrm{O}_{6} \mathrm{Si} 521.2357$, found: 521.2356; Anal. Calcd for $\mathrm{C}_{34} \mathrm{H}_{46} \mathrm{O}_{6} \mathrm{Si}: \mathrm{C}, 70.55 ; \mathrm{H}, 8.00$. Found: C, 70.61; H, 8.07.

\section{(+)-(3S,4R,5R,6R)-5-Benzyloxy-7-tert-butyl-diphenylsilyloxy-}

\section{2,2-dimethyl-3-methoxy-4,6-methylenedioxyheptanal. To a}

solution of the above alcohol $(0.613 \mathrm{~g}, 1.06 \mathrm{mmol})$ in DMSO $(8$ $\mathrm{mL})$ were added $\mathrm{Et}_{3} \mathrm{~N}(1.03 \mathrm{~mL}, 7.41 \mathrm{mmol})$ and $\mathrm{SO}_{3} \cdot \mathrm{Py}(0.505$ $\mathrm{g}, 3.18 \mathrm{mmol})$. The mixture was stirred for $2.5 \mathrm{~h}$, and quenched

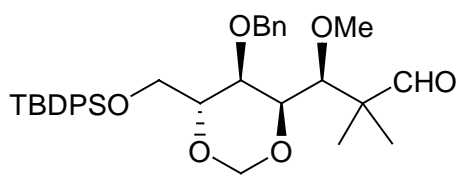
by $\mathrm{H}_{2} \mathrm{O}$. The resulting mixture was extracted with $\mathrm{Et}_{2} \mathrm{O}(100 \mathrm{~mL}$ x 3$)$. The combined organic extracts were washed with brine, dried over $\mathrm{MgSO}_{4}$ and concentrated. Purification of the crude product by flash chromatography (17\% EtOAc/hexane) provided the corresponding aldehyde $(0.593 \mathrm{~g}, 97 \%)$ as a colorless oil.

Data for aldehyde: $[\alpha]^{26}{ }_{\mathrm{D}}=+25.40^{\circ}\left(\mathrm{c} 1.10, \mathrm{CHCl}_{3}\right)$; IR (neat) $1722 \mathrm{~cm}^{-1} ;{ }^{1} \mathrm{H}$ NMR $(300 \mathrm{MHz}$, $\left.\mathrm{CDCl}_{3}\right) \delta 1.04(\mathrm{~s}, 3 \mathrm{H}), 1.08(\mathrm{~s}, 3 \mathrm{H}), 1.09(\mathrm{~s}, 9 \mathrm{H}), 3.46(\mathrm{~s}, 3 \mathrm{H}), 3.57(\mathrm{~d}, J=3.3 \mathrm{~Hz}, 1 \mathrm{H})$, 3.91-3.93 (m, $3 \mathrm{H}), 4.00(\mathrm{dd}, J=3.3,5.8 \mathrm{~Hz}, 1 \mathrm{H}), 4.06-4.11(\mathrm{~m}, 1 \mathrm{H}), 4.46(\mathrm{~d}, J=11.5 \mathrm{~Hz}, 1 \mathrm{H})$, $4.64(\mathrm{~d}, J=11.5 \mathrm{~Hz}, 1 \mathrm{H}), 4.75(\mathrm{~d}, J=5.5 \mathrm{~Hz}, 1 \mathrm{H}), 5.20$ (d, J=5.8 Hz,1 H), 7.26-7.43 (m, $11 \mathrm{H})$, 7.67-7.73 (m, $4 \mathrm{H}) ;{ }^{13} \mathrm{C} \mathrm{NMR}\left(75 \mathrm{MHz}, \mathrm{CDCl}_{3}\right) \delta 205.2,138.0,135.9,135.7,133.6,133.2,129.8$, 128.6, 128.1, 128.0, 127.8, 127.8, 90.0, 87.0, 75.2, 72.8, 71.3, 63.1, 61.2, 50.4, 26.8, 19.7, 19.4, 19.2, 15.2; LRMS $m / z 519\left(\mathrm{M}^{+}-57\right)$; HRMS calcd for $\mathrm{C}_{30} \mathrm{H}_{35} \mathrm{O}_{6} \mathrm{Si}$ 519.2200, found: 519.2203; Anal. Calcd for $\mathrm{C}_{34} \mathrm{H}_{44} \mathrm{O}_{6} \mathrm{Si}$ : C, 70.79; H, 7.68. Found: C, 70.90; H, 7.67.

(+)-(4S,5R,6R,7R)-6-Benzyloxy-8-tert-butyldiphenylsilylox y-3,3-dimethyl-4-methoxy-5,7-methylenedioxyoct-1-ene (25). To a solution of $\mathrm{Ph}_{3} \mathrm{P}^{+} \mathrm{CH}_{3} \mathrm{Br}^{-}(0.372 \mathrm{~g}, 1.04 \mathrm{mmol})$ in THF $(8 \mathrm{~mL})$ was added dropwise BuLi $(0.680 \mathrm{~mL}, 1.53 \mathrm{M}$ in

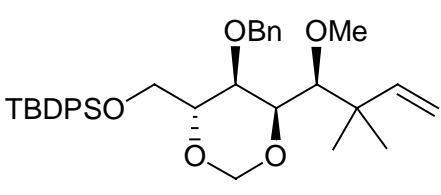

25 
hexane, $1.04 \mathrm{mmol}$ ). The solution was warmed to $65^{\circ} \mathrm{C}$, and stirred for $15 \mathrm{~min}$, and then cooled to $0{ }^{\circ} \mathrm{C}$. A solution of the above aldehyde $(0.200 \mathrm{~g}, 0.346 \mathrm{mmol})$ in THF $(2 \mathrm{~mL})$ was added at $0{ }^{\circ} \mathrm{C}$, and the mixture was stirred for 20 min. Saturated $\mathrm{NaHCO}_{3}(5 \mathrm{~mL})$ was added to quench the reaction and the resulting mixture was extracted with $\mathrm{Et}_{2} \mathrm{O}(60 \mathrm{~mL} \times 3)$. The combined organic extracts were washed with brine, dried over $\mathrm{MgSO}_{4}$ and concentrated. Purification of the crude product by flash chromatography (9\% EtOAc/hexane) provided olefin $25(0.199 \mathrm{~g}, 100 \%)$ as a white solid.

Data for 25: $\mathrm{mp} 86-87^{\circ} \mathrm{C}$; $[\alpha]^{22}{ }_{\mathrm{D}}=+9.42^{\circ}\left(\mathrm{c} 0.85, \mathrm{CHCl}_{3}\right)$; IR (neat) $1605 \mathrm{~cm}^{-1} ;{ }^{1} \mathrm{H} \mathrm{NMR}(300$ $\left.\mathrm{MHz}, \mathrm{CDCl}_{3}\right) \delta 1.00(\mathrm{~s}, 3 \mathrm{H}), 1.03(\mathrm{~s}, 3 \mathrm{H}), 1.08(\mathrm{~s}, 9 \mathrm{H}), 3.22(\mathrm{~d}, J=4.1 \mathrm{~Hz}, 1 \mathrm{H}), 3.45$ (s, $\left.3 \mathrm{H}\right)$, $3.77(\mathrm{dd}, J=4.4,6.0 \mathrm{~Hz}, 1 \mathrm{H}), 3.85-3.93(\mathrm{~m}, 2 \mathrm{H}), 3.97$ (t, $J=4.4 \mathrm{~Hz}, 1 \mathrm{H}), 4.15$ ( q, $J=5.8 \mathrm{~Hz}, 1 \mathrm{H})$, $4.47(\mathrm{~d}, J=11.8 \mathrm{~Hz}, 1 \mathrm{H}), 4.66$ (d, $J=11.8 \mathrm{~Hz}, 1 \mathrm{H}), 4.75(\mathrm{~d}, J=5.8 \mathrm{~Hz}, 1 \mathrm{H}), 4.92$ (dd, $J=1.6,10.7$ Hz, $1 \mathrm{H}), 4.95$ (dd, $J=1.6,17.6 \mathrm{~Hz}, 1 \mathrm{H}), 5.18$ (d, $J=5.8 \mathrm{~Hz}, 1 \mathrm{H}), 5.88$ ( dd, $J=10.7,17.6 \mathrm{~Hz}, 1 \mathrm{H})$, 7.31-7.43 (m, $11 \mathrm{H}), 7.66-7.71$ (m, $4 \mathrm{H}) ;{ }^{13} \mathrm{C} \mathrm{NMR}\left(75 \mathrm{MHz}, \mathrm{CDCl}_{3}\right) \delta$ 146.1, 138.2, 135.8, 135.7, $133.5,133.3,129.9,128.6,128.1,127.9,127.9,127.8,111.7,90.0,86.6,74.7,73.6,72.0$, 71.9, $62.8,61.2,42.1,26.8,25.3,21.8,1.9$; LRMS $m / z 517\left(\mathrm{M}^{+}-57\right)$; HRMS calcd for $\mathrm{C}_{31} \mathrm{H}_{37} \mathrm{O}_{5} \mathrm{Si}$ 517.2408, found: 517.2410.

$(1 R, 5 R, 6 R, 8 R S, 10 S)-8$-Acetoxy-5-(tert-butyldiphenylsilyloxy)methyl-9,9-dimethyl-10-methoxy-2,4,7-trioxabicyclo[4.4.0]decane (27). Ozone was bubbled through a $-78^{\circ} \mathrm{C}$ solution of olefin $26(54.0 \mathrm{mg}, 0.111 \mathrm{mmol})$ in $\mathrm{MeOH}(10 \mathrm{~mL})$ for $15 \mathrm{~min}$. After removal of excess ozone, $\mathrm{Me}_{2} \mathrm{~S}(0.041 \mathrm{~mL}, 0.558 \mathrm{mmol})$ was added

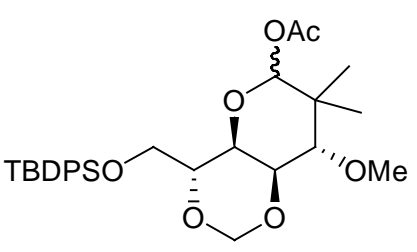

27 and the solution was stirred for $5 \mathrm{~h}$. The solution was concentrated to afford a clear oil (33.5 mg). Due to the instability of the crude lactol, it was immediately used without further purification.

A mixture of the crude lactol $(33.5 \mathrm{mg})$ and $\mathrm{Ac}_{2} \mathrm{O}(0.5 \mathrm{~mL})$ in pyridine $(1 \mathrm{~mL})$ was stirred for 16 h. The solvent was removed to leave a crude oil. Purification of the crude product by flash chromatography (14\% EtOAc/hexane) provided acetate 27 (58.0 mg, 99\% for 2 steps, as a 1.4:1 mixture of diastereoisomers) as a colorless oil.

Partial data for 27: IR (neat) $1745 \mathrm{~cm}^{-1} ;{ }^{1} \mathrm{H}$ NMR (300 MHz, $\left.\mathrm{CDCl}_{3}\right) \delta 0.97(\mathrm{~s}, 1.3 \mathrm{H}$ ), 0.98 (s, $1.7 \mathrm{H}), 1.07$ (s, $9 \mathrm{H}), 1.17(\mathrm{~s}, 1.7 \mathrm{H}), 1.21(\mathrm{~s}, 1.3 \mathrm{H}), 2.07$ (s, $1.3 \mathrm{H}), 2.10$ (s, $1.7 \mathrm{H}), 3.04$ (d, J=4.4 $\mathrm{Hz}, 0.4 \mathrm{H}), 3.23$ (d, J=3.6 Hz, 0.6 H), $3.42(\mathrm{~s}, 1.3 \mathrm{H}), 3.43(\mathrm{~s}, 1.7 \mathrm{H}), 3.81-4.21(\mathrm{~m}, 5 \mathrm{H}), 4.92(\mathrm{~d}$, $J=6.0 \mathrm{~Hz}, 0.4 \mathrm{H}), 4.96(\mathrm{~d}, J=6.0 \mathrm{~Hz}, 0.6 \mathrm{H}), 4.97(\mathrm{~d}, J=5.8 \mathrm{~Hz}, 0.4 \mathrm{H}), 5.08(\mathrm{~d}, J=5.8 \mathrm{~Hz}, 0.6 \mathrm{H})$, 5.71 (s, 0.6 H), 5.78 (s, 0.4 H), 7.26-7.47 (m, 6 H), 7.65-7.70 (m, 4 H); LRMS m/z $471\left(\mathrm{M}^{+}-57\right)$; HRMS calcd for $\mathrm{C}_{25} \mathrm{H}_{31} \mathrm{O}_{7} \mathrm{Si}$ 471.1837, found: 471.1835; Anal. Calcd for $\mathrm{C}_{29} \mathrm{H}_{40} \mathrm{O}_{7} \mathrm{Si}: \mathrm{C}, 65.88 ; \mathrm{H}$, 7.62. Found: C, 66.03; H, 7.62.

(+)-(1R,5R,6R,8R,10S)-5-(tert-Butyl-diphenylsilyloxy)methyl-9,9-dimethyl-10-methoxy8-(prop-2-enyl)-2,4,7-trioxabicyclo[4.4.0]decane. 
(+)-(1R,5R,6R,8R,10S)-5-(Hydroxy)methyl-10-methoxy-9,9-dimethyl -8-(prop-2-enyl)2,4,7-trioxabicyclo[4.4.0]decane (28).

A suspension of acetate 27 (10.4 mg, $0.0197 \mathrm{mmol})$ and activated $4 \AA$ molecular sieves $(0.1 \mathrm{~g})$ in $\mathrm{CH}_{3} \mathrm{CN}(5$ $\mathrm{mL}$ ) was stirred for $30 \mathrm{~min}$ at $0{ }^{\circ} \mathrm{C}$, and allyltrimethylsilane $(0.019 \mathrm{~mL}, 0118$ mmol) was added. The solution was
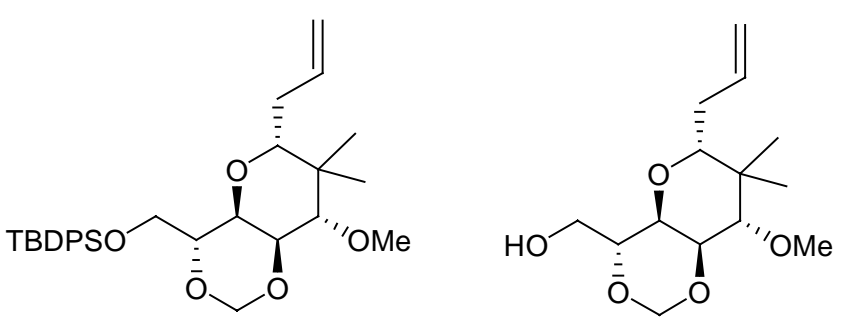

28 stirred for $20 \mathrm{~min}$ at $0{ }^{\circ} \mathrm{C}$, and $\mathrm{BF}_{3} \cdot \mathrm{Et}_{2} \mathrm{O}(7.0 \mu \mathrm{L}, 0.059 \mathrm{mmol})$ was added dropwise. The mixture was stirred for an additional $10 \mathrm{~min}$, and quenched by saturated $\mathrm{NaHCO}_{3}(5 \mathrm{~mL})$. After removal of $4 \AA$ molecular sieves through Celite, the filtrate was extracted with EtOAc $(3 \mathrm{x} 10 \mathrm{~mL})$. The combined organic extracts were washed with brine, dried over $\mathrm{MgSO}_{4}$ and concentrated to give a crude oil. The crude product was purified by flash chromatography. Elution with a 7:1 mixture of hexane-EtOAc afforded the corresponding silyl ether $(7.7 \mathrm{mg}, 77 \%)$ as a colorless oil. Elution with a 2:1 mixture of hexane-EtOAc afforded alcohol $28(0.9 \mathrm{mg}, 17 \%)$ as a colorless oil. Data for silyl ether: $[\alpha]^{26}{ }_{\mathrm{D}}=+75.70^{\circ}\left(\mathrm{c} 1.02, \mathrm{CHCl}_{3}\right)$; IR (neat) $1635 \mathrm{~cm}^{-1} ;{ }^{1} \mathrm{H} \mathrm{NMR}(300 \mathrm{MHz}$, $\left.\mathrm{CDCl}_{3}\right) \delta 0.87(\mathrm{~s}, 3 \mathrm{H}), 0.94(\mathrm{~s}, 3 \mathrm{H}), 1.30(\mathrm{~s}, 9 \mathrm{H}), 1.95-2.13(\mathrm{~m}, 2 \mathrm{H}), 2.98(\mathrm{dd}, J=2.6,9.5 \mathrm{~Hz}, 1$ H), 3.14 (d, J=10.2 Hz, $1 \mathrm{H}), 3.44$ (s, $3 \mathrm{H}), 3.90$ (ddd, $J=1.6,5.3,10.3 \mathrm{~Hz}, 1 \mathrm{H}), 3.99$ (dd, $J=5.5$, $11.3 \mathrm{~Hz}, 1 \mathrm{H}), 4.16(\mathrm{dd}, J=1.6,11.3 \mathrm{~Hz}, 1 \mathrm{H}), 4.23(\mathrm{dd}, J=6.9,10.4 \mathrm{~Hz}, 1 \mathrm{H}), 4.35$ (dd, $J=6.9$, $10.2 \mathrm{~Hz}, 1 \mathrm{H}), 4.77(\mathrm{~d}, J=6.6 \mathrm{~Hz}, 1 \mathrm{H}), 4.88(\mathrm{~d}, J=6.6 \mathrm{~Hz}, 1 \mathrm{H}), 5.07$ (dt, $J=9.3,0.9 \mathrm{~Hz}, 1 \mathrm{H}), 5.09$ (dt, $J=17.9,0.9 \mathrm{~Hz}, 1 \mathrm{H}), 5.87$ (ddt, $J=6.6,10.7,17.9 \mathrm{~Hz}, 1 \mathrm{H}), 7.28-7.79$ (m, $6 \mathrm{H}), 7.80-8.03$ (m, $4 \mathrm{H}) ;{ }^{13} \mathrm{C} \mathrm{NMR}\left(75 \mathrm{MHz}, \mathrm{CDCl}_{3}\right) \delta 136.2,135.9,135.9,129.8,129.8,127.8,116.5,87.2,79.7$, 78.4, 74.1, 73.5, 67.1, 63.8, 61.8, 41.5, 33.4, 26.8, 23.3, 19.3, 13.6; LRMS m/z $469\left(\mathrm{M}^{+}-41\right)$; HRMS calcd for $\mathrm{C}_{27} \mathrm{H}_{37} \mathrm{O}_{5} \mathrm{Si} 469.2408$, found: 469.2411; Anal. Calcd for $\mathrm{C}_{30} \mathrm{H}_{42} \mathrm{O}_{5} \mathrm{Si}$ : C, 70.54; H, 8.28. Found: C, 70.40; H, 8.05.

Data for 28: $[\alpha]^{27}{ }_{D}=+79.83^{\circ}\left(\mathrm{c} 1.77, \mathrm{CHCl}_{3}\right)$; IR (neat) $3458,1641,1178,912,841,613 \mathrm{~cm}^{-1} ;{ }^{1} \mathrm{H}$ NMR (400 MHz, $\left.\mathrm{CDCl}_{3}\right) \delta 0.89(\mathrm{~s}, 3 \mathrm{H}), 1.01(\mathrm{~s}, 3 \mathrm{H}), 2.00-2.08(\mathrm{~m}, 2 \mathrm{H}), 2.18$ (ddd, J=2.0, 6.9, $13.8 \mathrm{~Hz}, 1 \mathrm{H}), 3.28$ (dd, $J=2.2,10.4 \mathrm{~Hz}, 1 \mathrm{H}), 3.44$ (d, $J=10.2 \mathrm{~Hz}, 1 \mathrm{H}), 3.58$ (s, $3 \mathrm{H}), 3.65-3.71(\mathrm{~m}$, $1 \mathrm{H}), 3.82-3.88(\mathrm{~m}, 1 \mathrm{H}), 4.01-4.03(\mathrm{~m}, 2 \mathrm{H}), 4.16(\mathrm{dd}, J=6.4,10.2 \mathrm{~Hz}, 1 \mathrm{H}), 4.86$ (A of AB, $J=6.8$ $\mathrm{Hz}, 1 \mathrm{H}), 5.02$ (B of AB, J=6.8 Hz, $1 \mathrm{H}), 5.04-5.09$ (m, $2 \mathrm{H}), 5.73-5.83(\mathrm{~m}, 1 \mathrm{H}) ;{ }^{13} \mathrm{C}$ NMR $(75$ $\left.\mathrm{MHz}, \mathrm{CDCl}_{3}\right) \delta 135.9,117.1,86.9,78.5,73.5,72.9,68.4,63.4,61.7,41.6,33.4,23.1,13.1$; LRMS $\mathrm{m} / z 272\left(\mathrm{M}^{+}\right)$; Anal. Calcd for $\mathrm{C}_{14} \mathrm{H}_{24} \mathrm{O}_{5}$ : C, 61.74; H, 8.88. Found: C, 61.37; H, 8.63.

To a solution of the above products $(64 \mathrm{mg}$, a mixture of silyl ether and 28 ) in THF (5 mL) was added TBAF ( $0.10 \mathrm{~mL}, 1 \mathrm{M}$ in THF, $0.10 \mathrm{mmol})$. The solution was stirred for $6 \mathrm{~h}$ and saturated $\mathrm{NaHCO}_{3}(1 \mathrm{~mL})$ was added. The resulting mixture was extracted with EtOAc $(3 \mathrm{x} 7 \mathrm{~mL})$. The combined organic extracts were washed with brine, dried over $\mathrm{MgSO}_{4}$ and concentrated to give a crude oil. Purification of the crude product by flash chromatography (33\% EtOAc/hexane) provided alcohol 28 (25.8 $\mathrm{mg}, 86 \%$ for 2 steps) as a colorless oil. 
(+)-(1S,5R,6S,8R,10S)-10-Methoxy-5-\{ $N$-(methoxalyl)- $N$-[(2-trimethylsilyl)ethoxycarbonyl]amino\}-9,9-dimethyl-8-(prop-2-enyl)-2,4,7-trioxabic yclo[4.4.0]decane (30). To a $-78{ }^{\circ} \mathrm{C}$ mixture of carbamate 29 (111.6 mg, $278 \mu \mathrm{mol})$ and DMAP (44.2 mg, $362 \mu \mathrm{mol})$ in THF $(2.0 \mathrm{~mL})$ was added dropwise a solution of LHMDS in THF $(0.56$ $\mathrm{mL}, 1.0 \mathrm{M}, 0.56 \mathrm{mmol}$ ) over $10 \mathrm{~min}$. The resulting yellow solution was stirred for $0.5 \mathrm{~h}$, at which time a solution of methyl chlorooxoacetate in $\mathrm{CH}_{2} \mathrm{Cl}_{2}(0.14 \mathrm{~mL}, 3.0 \mathrm{M}, 0.42 \mathrm{mmol})$ was

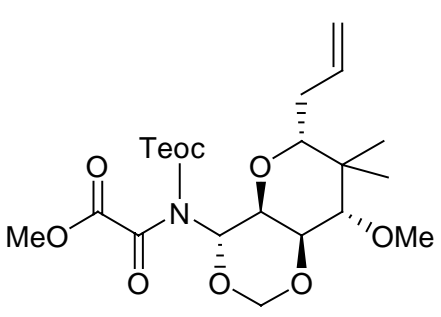

30 added dropwise. The mixture was stirred for $0.5 \mathrm{~h}$ at $-78{ }^{\circ} \mathrm{C}$, and warmed to $0{ }^{\circ} \mathrm{C}$, and stirred for 1 $\mathrm{h}$ at $0{ }^{\circ} \mathrm{C}$. The mixture was allowed to warm to rt, and poured into a slurry of $\mathrm{SiO}_{2}$ in hexane-EtOAc $(2: 1,10 \mathrm{~mL})$. The resulting suspension was vigorously stirred for $15 \mathrm{~min}$, and filtered through Celite. The filtrate was concentrated to give a crude oil. Purification of the crude product by chromatography (25\% EtOAc/hexane) provided imide 30 (105.8 mg, 78\%; $90 \%$ yield based on the recoverd 29) as a colorless oil.

Data for 30: $[\alpha]^{29}{ }_{\mathrm{D}}=+63.74^{\circ}\left(\mathrm{c} 1.80, \mathrm{CHCl}_{3}\right)$; IR (neat) 2955, 1714, 1715, 1541, 1252, 1177, 1128, 1109, 1084, 1030, 860, $839 \mathrm{~cm}^{-1} ;{ }^{1} \mathrm{H}$ NMR (300 MHz, $\mathrm{CDCl}_{3}$ referenced to $\left.7.24 \mathrm{ppm}\right) \delta$ 0.032 (s, $3 \mathrm{H}), 0.043$ (s, $6 \mathrm{H}), 0.86$ (s, $3 \mathrm{H}), 0.99$ (s, $3 \mathrm{H}), 1.08$ (dd,J=3.6, 6.0 Hz, $1 \mathrm{H}), 1.10$ (dd, J= 3.6, 6.0 Hz, $1 \mathrm{H}), 1.96-2.07$ (m, $1 \mathrm{H}), 2.10-2.18$ (m, $1 \mathrm{H}), 3.26$ (dd, J=2.0, $10.0 \mathrm{~Hz}, 1 \mathrm{H}), 3.44$ (d, $J=10.4 \mathrm{~Hz}, 1 \mathrm{H}), 3.56$ (s, $3 \mathrm{H}), 3.87$ (s, $3 \mathrm{H}), 4.30$ (dd, $J=7.2,10.8 \mathrm{~Hz}, 1 \mathrm{H}), 4.32$ ( dd, $J=3.6,6.0$ $\mathrm{Hz}, 1 \mathrm{H}), 4.34(\mathrm{dd}, J=3.2,6.0 \mathrm{~Hz}, 1 \mathrm{H}), 4.84(\mathrm{dd}, J=7.2,10.2 \mathrm{~Hz}, 1 \mathrm{H}), 4.90-5.03(\mathrm{~m}, 2 \mathrm{H}), 4.95$ (d, $J=6.8 \mathrm{~Hz}, 1 \mathrm{H}), 5.09(\mathrm{~d}, J=6.8 \mathrm{~Hz}, 1 \mathrm{H}), 5.66(\mathrm{tdd}, J=6.8,10.0,16.8 \mathrm{~Hz}, 1 \mathrm{H}), 6.10(\mathrm{~d}, J=10.4 \mathrm{~Hz}$, $1 \mathrm{H}) ;{ }^{13} \mathrm{C} \mathrm{NMR}\left(100 \mathrm{MHz}, \mathrm{CDCl}_{3}\right) \delta 162.6,161.0,152.2,135.6,116.3,87.6,79.4,78.8,77.3,75.1$, 67.5, 61.8, 52.9, 41.7, 33.6, 23.1, 17.4, 13.3, -1.4, -1.5, -1.5; LRMS m/z $446\left(\mathrm{M}^{+}-41\right.$, $\mathrm{CH}_{2} \mathrm{CH}=\mathrm{CH}_{2}$ ); HRMS calcd for $\mathrm{C}_{19} \mathrm{H}_{32} \mathrm{O}_{9} \mathrm{NSi} 446.1847$, found: 446.1845 .

(+)-(1S,5R,6S,8R,10S)-10-Methoxy-5-\{N-(methoxalyl)amino $\}-$ 9,9-dimethyl-8-(prop-2-enyl)-2,4,7-trioxabicyclo[4.4.0]decane

(3). To a $0{ }^{\circ} \mathrm{C}$ solution of imide $30(98.3 \mathrm{mg}, 0.202 \mathrm{mmol})$ in THF (3.0 mL) was added TBAF $(0.30 \mathrm{~mL}, 1.0 \mathrm{M}, 0.30 \mathrm{mmol})$. After 5 min, a mixture of $\mathrm{CH}_{2} \mathrm{Cl}_{2}(10 \mathrm{~mL})$ and $\mathrm{H}_{2} \mathrm{O}(10 \mathrm{~mL})$ was added. The aqueous phase was separated and further extracted with $\mathrm{CH}_{2} \mathrm{Cl}_{2}(2 \mathrm{x}$

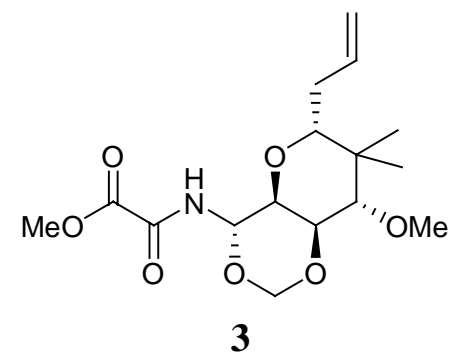
$10 \mathrm{~mL})$. The combined organic extracts were dried $\left(\mathrm{Na}_{2} \mathrm{SO}_{4}\right)$ and concentrated to afford a crude yellow solid. Purification of the crude product by flash chromatography (50\% EtOAc/hexane) provided methyl oxalate $3(64.5 \mathrm{mg}, 93 \%)$ as a white solid. The methyl oxalate $\mathbf{3}$ was used immediately in the next step.

Data for 3: $\mathrm{mp} 168-170{ }^{\circ} \mathrm{C}$; IR (KBr) 3315, 1738, 1701, 1541, 1263, 1182, 1123, 1103, 1076, 1036, 978, $907 \mathrm{~cm}^{-1} ;{ }^{1} \mathrm{H}$ NMR (400 MHz, $\mathrm{CDCl}_{3}$ referenced to $\left.7.24 \mathrm{ppm}\right) \delta 0.88(\mathrm{~s}, 3 \mathrm{H}), 1.02$ (s, $3 \mathrm{H}), 1.97-2.05$ (m, $1 \mathrm{H}), 2.16$ (dddd, $J=1.6,2.0,6.0,14.4 \mathrm{~Hz}, 1 \mathrm{H}), 3.29$ (dd, $J=2.0,10.0 \mathrm{~Hz}, 1 \mathrm{H}$ ), 3.46 (d, J=10.8 Hz, 1 H), 3.57 (s, 3 H), 3.91 (dd, J=6.8, $10.0 \mathrm{~Hz}, 1 \mathrm{H}), 3.93$ (s, $3 \mathrm{H}), 4.25$ (dd, J=6.4, 
$10.4 \mathrm{~Hz}, 1 \mathrm{H}$ ), 4.88 (d, $J=6.8 \mathrm{~Hz}, 1 \mathrm{H}), 4.88$ ( br d, $J=6.8 \mathrm{~Hz}, 1 \mathrm{H}$ ), 4.97 (dddd, $J=1.4,1.4,3.2$, $17.2 \mathrm{~Hz}, 1 \mathrm{H}), 5.15$ (d, $J=7.2 \mathrm{~Hz}, 1 \mathrm{H}), 5.63$ (tdd, $J=6.8,10.4,17.2 \mathrm{~Hz}, 1 \mathrm{H}), 5.74$ (dd, $J=10.0$, $10.0 \mathrm{~Hz}, 1 \mathrm{H}), 7.55(\mathrm{~d}, J=9.2 \mathrm{~Hz}, 1 \mathrm{H}) ;{ }^{13} \mathrm{C} \mathrm{NMR}\left(100 \mathrm{MHz}, \mathrm{CDCl}_{3}\right) \delta 160.0,156.2,135.5,116.2$, 86.7, 79.3, 78.7, 74.6, 74.1, 70.4, 61.8, 53.9, 41.7, 33.3, 23.1, 13.4; LRMS $m / z 344\left(\mathrm{M}^{+}+\mathrm{H}\right)$; HRMS calcd for $\mathrm{C}_{16} \mathrm{H}_{26} \mathrm{O}_{7} \mathrm{~N} 344.1709$, found : 344.1711 .

(2R,3R,4R)-2,3-Epoxy-4,5-isopropylidenedioxypentanol (36). To a -78 ${ }^{\circ} \mathrm{C}$ solution of methyl $(S)$-4,5-isoprorylidenedioxy-2-pentenoate $(39)^{1}$ (0.980 g, 4.91mmol) in THF (20 mL) was added DIBAL-H (10.3 mL, 1.0 $\mathrm{M}$ in hexane, $10.3 \mathrm{mmol})$. The mixture was stirred for $3.5 \mathrm{~h}$ at $-78{ }^{\circ} \mathrm{C}$, and

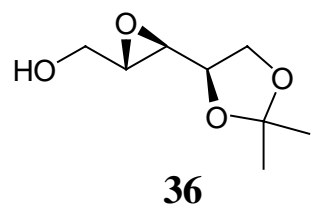
then $\mathrm{H}_{2} \mathrm{O}(10 \mathrm{~mL})$ was added. The solution was stirred for $30 \mathrm{~min}$ at $-78{ }^{\circ} \mathrm{C}$, at which time $\mathrm{Et}_{2} \mathrm{O}$ $(10 \mathrm{~mL})$ and hexane $(10 \mathrm{~mL})$ were added. The solution was allowed to warm to rt, and then filtered through Celite. The resulting clear solution was concentrated to afford a crude oil. Purification of the crude product by flash column chromatography $(50 \%$ EtOAc/hexane $)$ provided (S)-4,5-isopropylidenedioxy-2-pentenol $(0.682 \mathrm{~g}, 88 \%)$, in addition to recovered ester $39(0.110 \mathrm{~g}$, $11 \%)$. To a $-40{ }^{\circ} \mathrm{C}$ suspension of $\mathrm{Ti}\left(\mathrm{O}^{i} \mathrm{Pr}\right)_{4}(0.110 \mathrm{~mL}, 0.374 \mathrm{mmol})$ and powdered activated $3 \AA$ molecular sieves $(0.2 \mathrm{~g})$ in $\mathrm{CH}_{2} \mathrm{Cl}_{2}(5 \mathrm{~mL})$ was added a solution of (+)-DIPT $(0.087 \mathrm{~mL}, 0.416$ $\mathrm{mmol})$ in $\mathrm{CH}_{2} \mathrm{Cl}_{2}(2 \mathrm{~mL})$. The mixture was stirred for $40 \mathrm{~min}$ at $-40{ }^{\circ} \mathrm{C}$, and then a solution of (S)-4,5-isopropylidenedioxy-2-pentenol (0.658 g, $4.16 \mathrm{mmol})$ in $\mathrm{CH}_{2} \mathrm{Cl}_{2}(2 \mathrm{~mL})$ was added. After $1.5 \mathrm{~h}$, cumene hydroperoxide $(1.84 \mathrm{~mL}, 12.5 \mathrm{mmol})$ was added dropwise over $3 \mathrm{~min}$. The resulting solution was stirred for $89 \mathrm{~h}$ at $-40{ }^{\circ} \mathrm{C}$, and then cooled to $-78{ }^{\circ} \mathrm{C}$ and stirred for $10 \mathrm{~min} . \mathrm{Bu} 3 \mathrm{P}(2.09$ $\mathrm{mL}, 8.05 \mathrm{mmol}$ ) was added dropwise over $10 \mathrm{~min}$ to quench the reaction. The mixture was stirred for $30 \mathrm{~min}$, and then treated with citric acid monohydrate (ca. $87 \mathrm{mg}, 0.42 \mathrm{mmol}$ ) dissolved in acetone-ether $(1: 9,6 \mathrm{~mL})$. The cooling bath was removed, and the resulting mixture was stirred for an additional $40 \mathrm{~min}$. After filtration through Celite, the filtrate was dried over $\mathrm{MgSO}_{4}$ and concentrated to give an oil. Purification of the residue by flash chromatography (33\% EtOAc/hexanes) provided epoxide 36 ( $0.644 \mathrm{~g}, 89 \%)$. The structure of epoxide 36 was confirmed by comparison with reported spectral data ${ }^{2,3} ;[\alpha]^{23}{ }_{\mathrm{D}}=-21.36^{\circ}\left(\mathrm{c} 1.80, \mathrm{CHCl}_{3}\right)$, lit. ${ }^{4}[\alpha]_{\mathrm{D}}=-21.5^{\circ}$ (c $0.77, \mathrm{CHCl}_{3}$ ); IR (neat) 3435, 1637, 1376, 1217, $1058 \mathrm{~cm}^{-1} ;{ }^{1} \mathrm{H} \mathrm{NMR}\left(500 \mathrm{MHz}, \mathrm{CDCl}_{3}\right.$ referenced to $7.26 \mathrm{ppm}) \delta 1.37(\mathrm{~s}, 3 \mathrm{H}), 1.43(\mathrm{~s}, 3 \mathrm{H}), 1.89(\mathrm{dd}, J=5.5,7.3 \mathrm{~Hz}, 1 \mathrm{H}), 3.11$ (dd, $J=2.3$, $4.6 \mathrm{~Hz}, 1 \mathrm{H}), 3.15(\mathrm{td}, J=2.3,3.7 \mathrm{~Hz}, 1 \mathrm{H}), 3.68$ (ddd, $J=3.7,7.4,12.4 \mathrm{~Hz}, 1 \mathrm{H}), 3.83-3.88(\mathrm{~m}, 1 \mathrm{H})$, 3.95 (ddd, $J=2.3,5.0,12.8 \mathrm{~Hz}, 1 \mathrm{H}$ ), 4.09 (dt, $J=5.1,6.9 \mathrm{~Hz}, 1 \mathrm{H}), 4.11$ (dd, $J=6.4,13.8 \mathrm{~Hz}, 1 \mathrm{H}$ ); ${ }^{13} \mathrm{C}$ NMR $\left(100 \mathrm{MHz}, \mathrm{CDCl}_{3}\right) \delta 110.0,75.1,66.0,60.8,55.4,55.0,26.3,25.5$.

(1) Takano, S.; Kurotaki, A.; Takahashi, M.; Ogasawara, K. Synthesis 1986, 403.

(2) Katsuki, T.; Lee, A. W. M.; Ma, P.; Matrin, V. S.; Masamune, S.; Sharpless, K. B.; Tuddenham, D.; Walker, F. J. J. Org. Chem. 1982, 47, 1373.

(3) Scheuplein, S. W.; Kusche, A.; Bruckner, R.; Harms, K. Chem. Ber. 1990, 123, 917.

(4) Minami, N.; Ko, S. S.; Kishi, Y. J. Am. Chem. Soc. 1982, 104, 1109. 
(-)-(2S,3R,4R)-1-tert-Butyldiphenylsilyloxy-4,5-isopropylidenedioxy-2-mesyloxy-3-methylpentane (41). To a $0{ }^{\circ} \mathrm{C}$ solution of the above alcohols $(0.205 \mathrm{~g}, 0.479 \mathrm{mmol}$; a 7:1 mixture of $\mathbf{4 0}$ and the isomer) in $\mathrm{CH}_{2} \mathrm{Cl}_{2}(5 \mathrm{~mL})$ were added $\mathrm{Et}_{3} \mathrm{~N}(0.33 \mathrm{~mL}, 2.40 \mathrm{mmol})$ and $\mathrm{MsCl}(0.048 \mathrm{~mL}, 0.623 \mathrm{mmol})$. The mixture was stirred for 30 min, and then poured into saturated $\mathrm{NaHCO}_{3}$ solution $(10 \mathrm{~mL})$. The

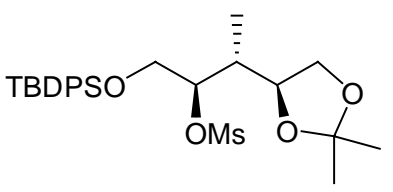

41 phases were separated and the aqueous phase was further extracted with $\mathrm{Et}_{2} \mathrm{O}(3 \times 15 \mathrm{~mL})$. The combined organic extracts were washed with brine $(10 \mathrm{~mL})$, dried $\left(\mathrm{MgSO}_{4}\right)$, and concentrated to give a yellow oil. The crude oil was chromatographed (11\% EtOAc/hexane), and then recrystallized twice from $\mathrm{Et}_{2} \mathrm{O}$ to yield methanesulfonate $41(0.168 \mathrm{~g}, 69 \%)$ as colorless prisms.

Data for 41: $\mathrm{mp} 113{ }^{\circ} \mathrm{C}$; $[\alpha]^{23}{ }_{\mathrm{D}}=-10.45^{\circ}\left(\mathrm{c} 0.60, \mathrm{CHCl}_{3}\right)$; IR $(\mathrm{KBr}) 1360,1176,1113,915,703$ $\mathrm{cm}^{-1} ;{ }^{1} \mathrm{H}$ NMR $\left(300 \mathrm{MHz}, \mathrm{CDCl}_{3}\right) \delta 0.90(\mathrm{~d}, J=7.2 \mathrm{~Hz}, 3 \mathrm{H}), 1.06(\mathrm{~s}, 9 \mathrm{H}), 1.30(\mathrm{~s}, 3 \mathrm{H}), 1.36(\mathrm{~s}$, $3 \mathrm{H}$ ), 2.18 (ddq, $J=10.9,6.8,3.8 \mathrm{~Hz}, 1 \mathrm{H}), 3.04$ (s, $3 \mathrm{H}), 3.59$ (dd, $J=6.3,7.4 \mathrm{~Hz}, 1 \mathrm{H}), 3.85$ (dd, $J=$ 3.2, 11.7 Hz, 1 H), 3.89-4.02 (m, $3 \mathrm{H}), 4.93(\mathrm{~m}, 1 \mathrm{H}), 7.40-7.48(\mathrm{~m}, 6 \mathrm{H}), 7.66-7.70(\mathrm{~m}, 4 \mathrm{H}) ;{ }^{13} \mathrm{C}$ NMR (75 MHz, $\left.\mathrm{CDCl}_{3}\right) \delta 135.6,135.5,132.9,132.7,130.0,127.9,109.1,85.7,76.2,67.8,63.7$, 39.5, 38.4, 26.7, 26.4, 19.0, 11.1; LRMS m/z $491\left(\mathrm{M}^{+}-15\right)$; Anal. Calcd for $\mathrm{C}_{26} \mathrm{H}_{38} \mathrm{O}_{6} \mathrm{SSi}$ : C, 61.63; H, 7.56; S, 6.33. Found: C, 61.53; H, 7.67; S, 6.41.

(-)-(2R,3R,4R)-4-(4-Methoxybenzyl)oxy-3-methylpentan-1,2-diol (42). To a solution of methanesulfonate $\mathbf{4 1}(1.23 \mathrm{~g}, 2.42 \mathrm{mmol})$ in THF $(10 \mathrm{~mL})$ was added TBAF (3.6 mL, 1.0 M in THF, $3.6 \mathrm{mmol})$. The mixture was stirred for $50 \mathrm{~min}$, and then saturated $\mathrm{K}_{2} \mathrm{CO}_{3}$ solution $(10 \mathrm{~mL})$ was added.

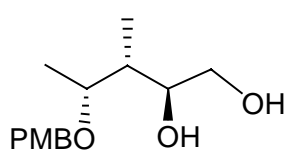

42 The mixture was stirred for an additional $10 \mathrm{~min}$. The phases were separated and the aqueous phase was further extracted with $\mathrm{Et}_{2} \mathrm{O}(3 \times 20 \mathrm{~mL})$. The combined organic extracts were washed with brine, dried $\left(\mathrm{MgSO}_{4}\right)$, and concentrated, providing the corresponding epoxide $(0.46 \mathrm{~g})$ which was used immediately in the next step.

To a $23{ }^{\circ} \mathrm{C}$ bath cooled suspension of $\mathrm{LiAlH}_{4}(0.184 \mathrm{~g}, 4.85 \mathrm{mmol})$ in $\mathrm{Et}_{2} \mathrm{O}(10 \mathrm{~mL})$ was added dropwise a solution of the crude epoxide in $\mathrm{Et}_{2} \mathrm{O}(2 \mathrm{~mL})$. The mixture was stirred for $12 \mathrm{~h}$ at $\mathrm{rt}$, and then cooled to $0{ }^{\circ} \mathrm{C}$, and $\mathrm{H}_{2} \mathrm{O}(0.18 \mathrm{~mL})$ was added. After $15 \mathrm{~min}, 15 \% \mathrm{NaOH}(0.18 \mathrm{~mL})$ and $\mathrm{H}_{2} \mathrm{O}$ $(0.55 \mathrm{~mL})$ were added and the resulting solution was stirred for an additional $2 \mathrm{~h}$ at rt. $\mathrm{MgSO}_{4}(0.5$ g) was added, and the solution was filterd through Celite and concentrated to afford a crude alcohol (0.42 g). This crude alcohol was immediately used without further purification.

To a $0{ }^{\circ} \mathrm{C}$ suspension of $\mathrm{NaH}(0.485 \mathrm{~g}, 12.1 \mathrm{mmol})$ in DMF $(5 \mathrm{~mL})$ was added a solution of the crude alcohol $(0.42 \mathrm{~g})$ in DMF $(2 \mathrm{~mL})$. The mixture was stirred for $1 \mathrm{~h}$ at $0{ }^{\circ} \mathrm{C}$, and warmed to rt, and then $\mathrm{PMBCl}(0.43 \mathrm{~mL}, 3.15 \mathrm{mmol})$ was added. The solution was stirred for $7 \mathrm{~h}$ at $\mathrm{rt}$, and then cooled to $0{ }^{\circ} \mathrm{C} . \mathrm{H}_{2} \mathrm{O}(5 \mathrm{~mL})$ was added slowly to quench the reaction. The resulting solution was extracted with $\mathrm{Et}_{2} \mathrm{O}(3 \times 15 \mathrm{~mL})$. The combined organic extracts were washed with brine $(15 \mathrm{~mL})$, dried $\left(\mathrm{MgSO}_{4}\right)$ and concentrated to afford a crude $p$-methoxybenzyl ether $(1.0 \mathrm{~g})$ which was 
dissolved in $\mathrm{AcOH}-\mathrm{THF}-\mathrm{H}_{2} \mathrm{O}(3: 1: 1,5 \mathrm{~mL})$. The mixture was warmed to $55^{\circ} \mathrm{C}$, and stirred for 9 h. After removal of the solvents by azeotropic distillation, the residue was purified by flash chromatography (50\% EtOAc/hexane) to provide diol 42 (0.482 g, 78\% from 41) as a colorless oil.

Data for 42: $[\alpha]^{24}{ }_{\mathrm{D}}=-10.00^{\circ}\left(\mathrm{c} 1.09, \mathrm{CHCl}_{3}\right)$; IR (neat) $3414,1613,1512,1246,1038,822,421$ $\mathrm{cm}^{-1}$; ${ }^{1} \mathrm{H}$ NMR $\left(300 \mathrm{MHz}, \mathrm{CDCl}_{3}\right) \delta 0.85(\mathrm{~d}, J=7.2 \mathrm{~Hz}, 3 \mathrm{H}), 1.23(\mathrm{~d}, J=6.3 \mathrm{~Hz}, 3 \mathrm{H}), 1.98-2.10$ (m, $1 \mathrm{H}), 2.25$ (br s, $1 \mathrm{H}), 3.51$ (dd, $J=5.7,11.4 \mathrm{~Hz}, 1 \mathrm{H}), 3.66-3.79(\mathrm{~m}, 3 \mathrm{H}), 3.81$ (s, $3 \mathrm{H}), 4.16$ (br s, $1 \mathrm{H}), 4.44(\mathrm{~d}, J=10.8 \mathrm{~Hz}, 1 \mathrm{H}), 4.55(\mathrm{~d}, J=10.8 \mathrm{~Hz}, 1 \mathrm{H}), 6.88(\mathrm{~d}, J=8.7 \mathrm{~Hz}, 2 \mathrm{H}), 7.25(\mathrm{~d}, J=$ $8.7 \mathrm{~Hz}, 2 \mathrm{H}) ;{ }^{13} \mathrm{C} \mathrm{NMR}\left(75 \mathrm{MHz}, \mathrm{CDCl}_{3}\right) \delta 159.4,130.1,129.3,113.9,77.6,70.2,64.8,55.1,37.8$, 14.4, 12.2; LRMS $m / z 254\left(\mathrm{M}^{+}\right)$; HRMS calcd for $\mathrm{C}_{14} \mathrm{H}_{22} \mathrm{O}_{4} 254.1518$, found: 254.1514.

(+)-(3R,4R)-4-(4-Methoxybenzyl)oxy-3-methylpentene (43). To a mixture of diol $42(1.46 \mathrm{~g}, 5.75 \mathrm{mmol})$ and $\mathrm{K}_{2} \mathrm{CO}_{3}(3.18 \mathrm{~g}, 23.0 \mathrm{mmol})$ in benzene $(50 \mathrm{~mL})$ was added $\mathrm{Pb}(\mathrm{OAc})_{4}(5.61 \mathrm{~g}, 12.6 \mathrm{mmol})$. The mixture was stirred for $1.5 \mathrm{~h}$ and then filtered through Celite. The filtrate was concentrated to give a colorless oil which was used immediately in the next step.

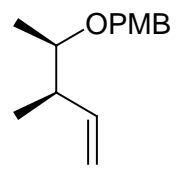

43

To a $0{ }^{\circ} \mathrm{C}$ suspension of $\mathrm{MePPh}_{3}{ }^{+} \mathrm{Br}^{-}(3.08 \mathrm{~g}, 8.62 \mathrm{mmol})$ in THF $(20 \mathrm{~mL})$ was added dropwise BuLi (6.48 mL, 1.33 M in hexane, $8.62 \mathrm{mmol}$ ) over $5 \mathrm{~min}$. The resulting yellow solution was warmed to rt and stirred for $20 \mathrm{~min}$. After the yellow solids had dissipated, a solution of the crude aldehyde in THF $(10 \mathrm{~mL})$ was added at $0{ }^{\circ} \mathrm{C}$. The solution was allowed to warm to rt and stirred for 2 h. Saturated $\mathrm{NH}_{4} \mathrm{Cl}(15 \mathrm{~mL})$ solution was added to quench the reaction. The phases were separated and the aqueous phase was further extracted with EtOAc $(3 \times 50 \mathrm{~mL})$. The combined organic extracts were washed with brine, dried $\left(\mathrm{MgSO}_{4}\right)$ and concentrated to give a crude oil. Purification of the crude oil by flash chromatography (5\% EtOAc/hexane) provided alkene $\mathbf{4 3}$ (0.834 g, $69 \%$ for 2 steps) as a colorless oil.

Data for 43: $[\alpha]^{29}{ }_{D}=+2.20^{\circ}\left(\mathrm{c} 1.16, \mathrm{CHCl}_{3}\right)$; IR (neat) $1614,1514,1456,1248,1092,1038,822$ $\mathrm{cm}^{-1}$; ${ }^{1} \mathrm{H}$ NMR (400 MHz, $\mathrm{CDCl}_{3}$ referenced to $\left.7.24 \mathrm{ppm}\right) \delta 7.26(\mathrm{td}, J=2.5,8.4 \mathrm{~Hz}, 2 \mathrm{H}), 6.86(\mathrm{td}$, $J=2.8,8.4 \mathrm{~Hz}, 2 \mathrm{H}), 5.81$ (ddd, $J=7.6,10.8,18.0 \mathrm{~Hz}, 1 \mathrm{H}), 5.04$ (ddd, $J=1.6,2.0,17.6 \mathrm{~Hz}, 1 \mathrm{H}$ ), 5.01 (ddd, $J=1.2,2.0,10.0 \mathrm{~Hz}, 1 \mathrm{H}), 4.51$ (d, $J=11.2 \mathrm{~Hz}, 1 \mathrm{H}), 4.41$ (d, J=11.2 Hz, $1 \mathrm{H}$ ), 3.80 (s, 3 H), 3.34 (qd, $J=6.4,12.4 \mathrm{~Hz}, 1 \mathrm{H}), 2.36$ (ddq, $J=6.8,6.8,6.8 \mathrm{~Hz}, 1 \mathrm{H}), 1.12$ (d, J=6.0 Hz, $3 \mathrm{H}$ ), $1.03(\mathrm{~d}, J=6.8 \mathrm{~Hz}, 3 \mathrm{H}) ;{ }^{13} \mathrm{C} \mathrm{NMR}\left(100 \mathrm{MHz}, \mathrm{CDCl}_{3}\right) \delta 158.9,140.7,131.0,129.0,114.3,114.0$, 78.1, 70.3, 55.2, 43.0, 16.7; LRMS m/z $220\left(\mathrm{M}^{+}\right)$; Anal. Calcd for $\mathrm{C}_{14} \mathrm{H}_{20} \mathrm{O}_{2}: \mathrm{C}, 76.33 ; \mathrm{H}, 9.15$. Found: C, 76.45; H, 9.09.

(2RS)-2-[(1R,2R)-2-Hydroxy-1-methylpropyl]cyclopropane-1,1-dimethyl diazocarboxylate. To a solution of cyclopropane $46(0.395 \mathrm{~g}, 1.13 \mathrm{mmol})$ in $\mathrm{MeCN}_{-} \mathrm{H}_{2} \mathrm{O}(10: 1 \mathrm{v} / \mathrm{v}, 10 \mathrm{~mL})$ was added ceric ammonium nitrate $(1.86 \mathrm{~g}, 3.39 \mathrm{mmol})$ in one portion. After $2 \mathrm{~h}$, the reaction was quenched by the addition of saturated $\mathrm{NaHCO}_{3}(15 \mathrm{~mL})$ and $\mathrm{NaHSO}_{3}(0.4 \mathrm{~g})$. The mixture was stirred vigorously for a further $15 \mathrm{~min}$ and then filtered. The filter cake was washed several times 
$1135 \mathrm{~cm}^{-1} ;{ }^{1} \mathrm{H}$ NMR $\left(400 \mathrm{MHz}, \mathrm{CDCl}_{3}\right) \delta$ 0.98-1.03 (m, 1H), $1.01(\mathrm{~d}, J=5.2 \mathrm{~Hz}, 3 \mathrm{H}), 1.22(\mathrm{~d}$, $J=6.4 \mathrm{~Hz}, 3 \mathrm{H}), 1.26(\mathrm{t}, J=7.2 \mathrm{~Hz}, 3 \mathrm{H}), 1.39$ (dd, $J=4.8$ and $9.2 \mathrm{~Hz}, 1 \mathrm{H}), 1.53$ (dd, $J=4.4$ and $8.0 \mathrm{~Hz}$, $1 \mathrm{H}), 1.85-1.94(\mathrm{~m}, 1 \mathrm{H}), 3.78-3.87(\mathrm{~m}, 1 \mathrm{H}), 4.09-4.30(\mathrm{~m}, 4 \mathrm{H}) ;{ }^{13} \mathrm{C} \mathrm{NMR}\left(100 \mathrm{MHz}, \mathrm{CDCl}_{3}\right) \delta$ $170.4,168.4,71.1,61.3,61.3,39.6,33.5,32.2$, 20.8, 20.6, 14.0, 13.9, 13.8; LRMS $\mathrm{m} / z 259$ $\left(\mathrm{M}^{+}+1\right)$; HRMS calcd for $\mathrm{C}_{13} \mathrm{H}_{29} \mathrm{O}_{5} 259.1545$, found: 259.1549 .

(4S,5R,6R)-5,6-Dimethyl-4-(Phenylselenylmethyl)tetrahydro-2H-pryan-2-one. $\quad$ To a suspension of diphenyldiselenide $(570 \mathrm{mg}, 1.84 \mathrm{mmol})$ in $\mathrm{EtOH}(5 \mathrm{~mL})$ was added $\mathrm{NaBH}_{4}$ (34.9 mg, $0.923 \mathrm{mmol}$ ) at rt. After the yellow color dissipated, a solution of alcohol 44 (106 mg, $0.460 \mathrm{mmol})$ in EtOH $(2 \mathrm{~mL})$ was added. The mixture was heated for $12 \mathrm{~h} .10 \% \mathrm{HCl}$ solution $(10 \mathrm{~mL})$ was added and the resulting mixture

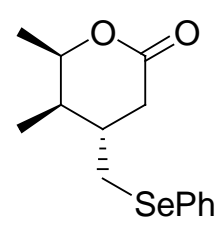
was stiired for $5 \mathrm{~min}$. After addition of EtOAc, the mixture was separated. The aqueous layer was extracted twice with EtOAc, the combined organic layers were wached with brine, dried $\left(\mathrm{MgSO}_{4}\right)$, and concentrated under reduced pressure. The crude product was used in the next step without purification.

A mixture of the above product and $\mathrm{LiI}(73.5 \mathrm{mg}, 0.548 \mathrm{mmol})$ in DMF $(5 \mathrm{~mL})$ was refluxed for $15 \mathrm{~h} . \mathrm{Et}_{2} \mathrm{O}(10 \mathrm{~mL})$ and water $(10 \mathrm{~mL})$ were added, and the resulting mixture was separated. The aqueous layer was extracted twice with $\mathrm{Et}_{2} \mathrm{O}$. The combined organic layers were washed with water, brine, dried $\left(\mathrm{MgSO}_{4}\right)$, and concentrated. Purification of the crude product by flash chromatography (30\% EtOAc/hexane) gave the desired $\alpha$-phenylselenylmethyl $\delta$-lactone (71.4 $\mathrm{mg}, 52 \%)$ as a yellow oil.

${ }^{1} \mathrm{H}$ NMR (300 MHz) $\delta$ 7.54-7.48 (2H, m), 7.32-7.25 (3H, m), 4.56-4.4.6 (1H, m), 3.08-2.92 (2H, m), $2.68(1 \mathrm{H}, \mathrm{dd}, J=5.9,15.5 \mathrm{~Hz}), 2.38(1 \mathrm{H}, \mathrm{dd}, J=8.6,15.5 \mathrm{~Hz}), 2.00-1.84(2 \mathrm{H}, \mathrm{m}), 1.29(3 \mathrm{H}, \mathrm{d}$, $J=6.9 \mathrm{~Hz}), 0.95(3 \mathrm{H}, \mathrm{d}, J=6.9 \mathrm{~Hz}) .{ }^{13} \mathrm{C}$ NMR $(75 \mathrm{~Hz}) \delta 171.9,132.9,129.3,129.2,127.3,75.8$, 37.4, 36.9, 34.3, 33.8, 17.0, 13.9. LRMS $m / z 298\left(\mathrm{M}^{+}\right)$; HRMS calcd for $\mathrm{C}_{14} \mathrm{H}_{18} \mathrm{O}_{2} \mathrm{Se} 298.0472$, found 298.0474 .

(4R,5R,6R)-5,6-Dimethyl-4-(Phenylselenylmethyl)tetrahydro-2H-pryan-2-one. $\quad$ To a suspension of diphenyldiselenide $(168 \mathrm{mg}, 0.538 \mathrm{mmol})$ in $\mathrm{EtOH}(3 \mathrm{~mL})$ was added $\mathrm{NaBH}_{4}(21.0 \mathrm{mg}, 0.555 \mathrm{mmol})$ at rt. After the yellow color dissipated, a solution of alcohol 45 (31 mg, $0.135 \mathrm{mmol})$ in $\mathrm{EtOH}(2 \mathrm{~mL})$ was added. The mixture was heated for $16 \mathrm{~h} .10 \% \mathrm{HCl}$ solution $(5 \mathrm{~mL})$ was added and the resulting mixture was stiired for $15 \mathrm{~min}$. After addition of EtOAc, the mixture

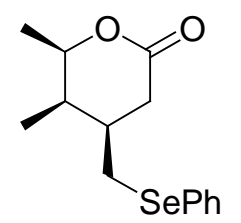
was separated. The aqueous layer was extracted twice with EtOAc, the combined organic layers were wached with brine, dried $\left(\mathrm{MgSO}_{4}\right)$, and concentrated under reduced pressure. The crude product was used in the next step without purification.

A mixture of the above product and LiI (45.2 mg, $0.337 \mathrm{mmol}$ ) in DMF (4 mL) was refluxed for 11 h. $\mathrm{Et}_{2} \mathrm{O}(8 \mathrm{~mL})$ and water $(8 \mathrm{~mL})$ were added, and the resulting mixture was separated. The aqueous layer was extracted twice with $\mathrm{Et}_{2} \mathrm{O}$. The combined organic layers were washed with 
water, brine, dried $\left(\mathrm{MgSO}_{4}\right)$, and concentrated. Purification of the crude product by flash chromatography (30\% EtOAc/hexane) gave the desired $\beta$-phenylselenylmethyl $\delta$-lactone (17.7 $\mathrm{mg}, 44 \%)$ as a yellow oil. ${ }^{1} \mathrm{H}$ NMR spectrum of this compound agrees with the literature. ${ }^{1}$

${ }^{1} \mathrm{H}$ NMR (300 MHz) $\delta 7.56-7.44(2 \mathrm{H}, \mathrm{m}), 7.32-7.19(3 \mathrm{H}, \mathrm{m}), 4.44(1 \mathrm{H}, \mathrm{dq}, J=2.1,6.9 \mathrm{~Hz})$, 2.94-2.66 (3H, m), 2.32-2.00 (3H, m), $1.33(3 \mathrm{H}, \mathrm{d}, J=6.9 \mathrm{~Hz}), 0.84(3 \mathrm{H}, \mathrm{d}, J=6.9 \mathrm{~Hz})$.

(1) Kocienski, P.; Jarowicki, K.; Marczak. Synthesis 1991, 1191.

\section{(2R,3R,4RS)-3,4-Dihydro-2,3-dimethyl-4-phenylselenylmethyl-}

6-trimethylstannyl-2H-pyran (2). To a $-78{ }^{\circ} \mathrm{C}$ solution of lactones 31 $(114.3 \mathrm{mg}, 0.384 \mathrm{mmol})$ in THF $(2.5 \mathrm{~mL})$ was added dropwise KHMDS (1.00 mL, 0.5 M in toluene, $0.500 \mathrm{mmol}$ ) over $20 \mathrm{~min}$. After $15 \mathrm{~min}$, HMPA $(0.100 \mathrm{~mL}, 0.575 \mathrm{mmol})$ was added and the mixture was stirred for $2 \mathrm{~h}$ at the same temperature. A solution of $\operatorname{PhNTf}_{2}(0.164 \mathrm{mg}, 0.460 \mathrm{mmol}$,

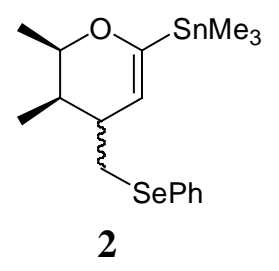
recrystallized from $\mathrm{CH}_{2} \mathrm{Cl}_{2}$-hexane) in THF ( $2 \mathrm{~mL}$ ) was added dropwise and the mixture was stirred at $0{ }^{\circ} \mathrm{C}$ for $1 \mathrm{~h}$ and at $\mathrm{rt}$ for $2 \mathrm{~h}$. The solvent was removed to give the oily residue, which was dissolved in $\mathrm{Et}_{2} \mathrm{O}(10 \mathrm{~mL})$ and $\mathrm{H}_{2} \mathrm{O}(10 \mathrm{~mL})$. The separated aqueous phase was extracted with $\mathrm{Et}_{2} \mathrm{O}(3 \times 20 \mathrm{~mL})$. The combined organic extracts were washed with brine $(10 \mathrm{~mL})$, dried $\left(\mathrm{MgSO}_{4}\right)$ and concentrated to afford the crude enol triflate $(163 \mathrm{mg})$, which was used immediately in the next step.

To a solution of the crude enol triflate $(163 \mathrm{mg})$ in THF $(15 \mathrm{~mL})$ was added a solution of $\left(\mathrm{Me}_{3} \mathrm{Sn}\right)_{2}$ in THF $(0.580 \mathrm{~mL}, 1.0 \mathrm{M}, 0.580 \mathrm{mmol})$ followed by $\mathrm{Pd}\left(\mathrm{PPh}_{3}\right)_{4}(22.2 \mathrm{mg}, 19.2 \mu \mathrm{mol})$ and $\mathrm{LiCl}$ (162.6 mg, $3.84 \mathrm{mmol})$. The mixture was stirred under reflux for $12 \mathrm{~h}$. Saturated $\mathrm{NaHCO}_{3}$ solution $(10 \mathrm{~mL})$ was added to the mixture and the phases were separated. The aqueous phase was further extracted with $\mathrm{Et}_{2} \mathrm{O}(3 \times 20 \mathrm{~mL})$. The combined organic extracts were washed with brine $(20 \mathrm{~mL})$, dried $\left(\mathrm{MgSO}_{4}\right)$ and concentrated to give a crude oil. Purification of the crude oil by chromatography on alumina deactivated by $5 \%$ water eluting with petroleum ether provided dihydropyran $2(143.5 \mathrm{mg}, 84 \%)$ as a colorless oil.

Data for $2(\mathrm{dr}=3: 1)$ : IR(neat) 2970, 2922, 2876, 1603, 1578, 1477, 1437, 1379, 1252, 1070, 1022 ,

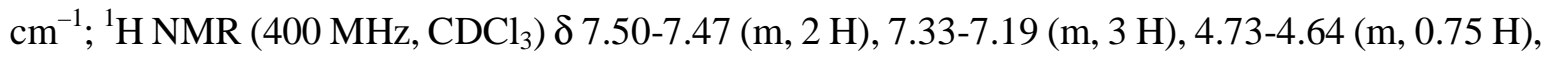
4.49-4.41 (m, 0.25 H), 3.95 (dq, $J=1.6,6.4$ Hz, 0.25 H), 3.78 (dq, J=2.4, 6.4 Hz, 0.75 H), 2.96 (dd, $J=6.0,12.4 \mathrm{~Hz}, 1 \mathrm{H}), 2.84(\mathrm{dd}, J=8.0,11.2 \mathrm{~Hz}, 0.75+0.25 \times 2 \mathrm{H}), 2.73-2.66(\mathrm{~m}, 0.25 \mathrm{H})$, 1.93-1.88 (m, $0.75 \mathrm{H}), 1.85-1.77(\mathrm{~m}, 1 \mathrm{H}), 1.18(\mathrm{~d}, J=6.0 \mathrm{~Hz}, 0.25$ x $3 \mathrm{H}), 1.14$ (d, J=6.8 Hz, 0.75 x $3 \mathrm{H}), 0.82(\mathrm{~d}, J=7.2 \mathrm{~Hz}, 0.75$ x $3 \mathrm{H}), 0.75(\mathrm{~d}, J=6.8 \mathrm{~Hz}, 0.25$ x $3 \mathrm{H}), 0.13(\mathrm{~s}, 9 \mathrm{H}) ;{ }^{13} \mathrm{C}$ NMR (100 $\left.\mathrm{MHz}_{\mathrm{CDCl}}\right) \delta 162.5,132.6,132.5,130.6,129.0,128.9,126.7,125.4,112.6,75.6,70.6,39.8$, $38.4,35.1,34.8,34.3,34.0,31.5,30.4,18.6,17.5,13.1,5.3,-9.6$; LRMS $m / z 446\left(\mathrm{M}^{+}\right)$; HRMS calcd for $\mathrm{C}_{17} \mathrm{H}_{26} \mathrm{OSeSn} 446.0171$, found: 446.0175 .

Data for the major isomer of 2 ( $4 \alpha$-isomer): $\mathrm{mp}<30{ }^{\circ} \mathrm{C}$; $[\alpha]^{26}{ }_{\mathrm{D}}=+51.86^{\circ}\left(\mathrm{c} 1.10, \mathrm{CHCl}_{3}\right)$; IR (neat) 2970, 2920, 2876, 1578, 1477, 1456, 1437, 1379, 1252, 1070, 1022, $\mathrm{cm}^{-1}$; ${ }^{1} \mathrm{H}$ NMR (400

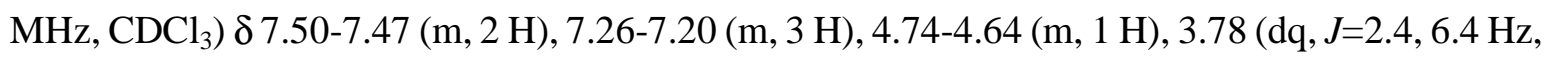


$1 \mathrm{H}), 2.97$ (dd, $J=6.0,12.4 \mathrm{~Hz}, 1 \mathrm{H}), 2.84$ (dd, $J=8.8,12.0 \mathrm{~Hz}, 1 \mathrm{H}), 1.93-1.88$ (m, $1 \mathrm{H}$ ), 1.84-1.77 $(\mathrm{m}, 1 \mathrm{H}), 1.14(\mathrm{~d}, J=6.4 \mathrm{~Hz}, 3 \mathrm{H}), 0.82(\mathrm{~d}, J=6.8 \mathrm{~Hz}, 3 \mathrm{H}), 0.13(\mathrm{~s}, 9 \mathrm{H}) ;{ }^{13} \mathrm{C}$ NMR $(100 \mathrm{MHz}$, $\left.\mathrm{CDCl}_{3}\right) \delta 162.4,132.6,130.6,128.9,126.7,125.4,112.6,70.6,39.8,35.1,34.8,30.4,17.5,13.1$, -9.7 .

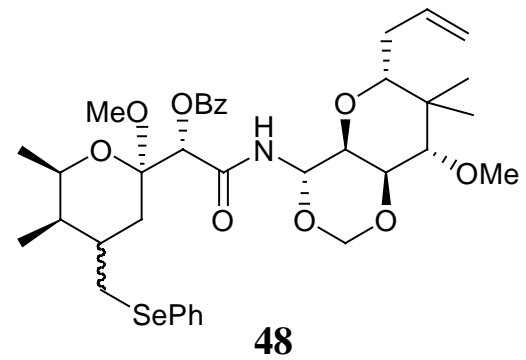

(4RS)-7-Benzoyl-4-phenylselenylmethyl-15-(prop-2-enyl)-mycalamide A (48). To a $-90{ }^{\circ} \mathrm{C}$ solution of ketone $47(21.3 \mathrm{mg}, 35.9 \mu \mathrm{mol})$ in $(1.5 \mathrm{~mL})$ was added dropwise $\mathrm{LiBH}\left({ }^{s} \mathrm{Bu}\right)_{3}(0.47 \mathrm{~mL}$, $0.23 \mathrm{M}$ in THF, $0.108 \mathrm{mmol}$ ) over $20 \mathrm{~min}$. After stirring at $-90{ }^{\circ} \mathrm{C}$ for $15 \mathrm{~min}$, the reaction was quenched by the addition of brine $(3 \mathrm{~mL})$ and $\mathrm{CH}_{2} \mathrm{Cl}_{2}(3 \mathrm{~mL})$. The mixture was stirred vigorously for a further $20 \mathrm{~min}$. The separated aqueous phase was extracted with $\mathrm{CH}_{2} \mathrm{Cl}_{2}(10 \mathrm{~mL} \times 3)$, dried $\left(\mathrm{Na}_{2} \mathrm{SO}_{4}\right)$ and concentrated to give a colorless oil, which was used immediately in the next step. The residue was dissolved in a mixture of $\mathrm{CH}_{2} \mathrm{Cl}_{2}(2.3 \mathrm{~mL})$ and $\mathrm{MeOH}(0.2 \mathrm{~mL})$, and then CSA (2 $\mathrm{mg}$ ) was added. The mixture was stirred for $18 \mathrm{~h}$ before $\mathrm{K}_{2} \mathrm{CO}_{3}(8 \mathrm{mg})$ was added. The solution was then stirred for 40 min and poured onto saturated $\mathrm{NaHCO}_{3}$ solution $(3 \mathrm{~mL})$. The phases were separated and the aqueous phase was further extracted with $\mathrm{CH}_{2} \mathrm{Cl}_{2}(10 \mathrm{~mL} \times 3)$. The combined organic extracts were dried $\left(\mathrm{Na}_{2} \mathrm{SO}_{4}\right)$ and concentrated to give a yellow oil, which was used immediately in the next step. To a solution of the crude oil in $\mathrm{CH}_{2} \mathrm{Cl}_{2}(1 \mathrm{~mL})$ were added DMAP (8.8 mg, $72 \mu \mathrm{mol}),{ }^{i} \operatorname{Pr}_{2} \mathrm{NEt}(63 \mu \mathrm{L}, 0.36 \mathrm{mmol})$ and benzoyl chloride $(12 \mu \mathrm{L}, 0.10 \mathrm{mmol})$. The mixture was stirred for $1 \mathrm{~h}$ at $\mathrm{rt}$, and then $\mathrm{MeOH}(0.05 \mathrm{~mL})$ was added. After stirring for $10 \mathrm{~min}$, brine $(3 \mathrm{~mL})$ was added and the mixture was extracted with $\mathrm{CH}_{2} \mathrm{Cl}_{2}(10 \mathrm{~mL} \times 3)$. The combined organic extracts were dried $\left(\mathrm{Na}_{2} \mathrm{SO}_{4}\right)$ and concentrated to give a yellow solid. Purification of the crude solid by flash chromatography ( $33 \%$ EtOAc/hexane) provided the corresponding benzoates (19.8 $\mathrm{mg}, 75 \%$ for 3 steps, a mixture of 5 diastereoisomers) as a colorless solid. The diastereoisomers were separated by column chromatography (33\% EtOAc/hexane) to give the title compounds 48 (11.0 mg, a 5.4:1 mixture of diastereoisomers at C4, $42 \%$ for 3 steps).

Data for $48(\mathrm{dr}=13: 1)$ : $\mathrm{mp} 70-75^{\circ} \mathrm{C}$; $\mathrm{IR}(\mathrm{KBr}) 3362,1732,1699,1522,1269,1107,1034,739$, $710 \mathrm{~cm}^{-1} ;{ }^{1} \mathrm{H}$ NMR $\left(600 \mathrm{MHz}, \mathrm{C}_{6} \mathrm{D}_{6}\right.$ referenced to $\left.7.16 \mathrm{ppm}\right) \delta 8.31$ (ddd, $J=1.8,1.8,7.2 \mathrm{~Hz}, 2 \mathrm{H}$ ), 7.50-7.48 (m, 0.93 x $2 \mathrm{H}), 7.48-7.46$ (m, 0.07 x $2 \mathrm{H}), 7.31$ (d, J=9.6 Hz, 0.93 H), 7.06-6.91 (m, 6 $\mathrm{H}), 6.08-6.02(\mathrm{~m}, 0.07 \mathrm{H}), 6.00-5.95(\mathrm{~m}, 0.93 \mathrm{H}), 5.94(\mathrm{~s}, 0.07 \mathrm{H}), 5.93(\mathrm{t}, J=10.2 \mathrm{~Hz}, 0.07 \mathrm{H})$, $5.93(\mathrm{t}, J=10.2 \mathrm{~Hz}, 0.93 \mathrm{H}), 5.89$ (s, $0.93 \mathrm{H}), 4.97$ (dddd, $J=1.8,1.8,3.6,6.7 \mathrm{~Hz}, 0.93 \mathrm{H}), 4.95$ (dd, $J=1.2,2.1 \mathrm{~Hz}, 0.93 \mathrm{H}), 4.60(\mathrm{~d}, J=7.2 \mathrm{~Hz}, 0.07 \mathrm{H}), 4.58(\mathrm{~d}, J=7.2 \mathrm{~Hz}, 0.93 \mathrm{H}), 4.54(\mathrm{~d}, J=7.2 \mathrm{~Hz}$, $0.07 \mathrm{H}), 4.52(\mathrm{~d}, J=7.2 \mathrm{~Hz}, 0.93 \mathrm{H}), 4.32$ (dd, $J=6.8,10.3 \mathrm{~Hz}, 1 \mathrm{H}), 4.29$ (dd, J=6.8, $10.4 \mathrm{~Hz}, 1 \mathrm{H}$ ), 3.77 (dd, $J=6.6,10.2 \mathrm{~Hz}, 0.93 \mathrm{H}), 3.73(\mathrm{dq}, J=2.4,6.6 \mathrm{~Hz}, 0.93 \mathrm{H}), 3.81(\mathrm{dd}, J=6.8,9.7 \mathrm{~Hz}, 0.07$ 
H), 3.56-3.54 (m, $0.07 \times 2 \mathrm{H}), 3.54(\mathrm{dd}, J=3.0,10.2 \mathrm{~Hz}, 0.93 \mathrm{H}), 3.31(\mathrm{dd}, J=7.2,12.6 \mathrm{~Hz}, 0.93 \mathrm{H})$, 3.27 (s, $0.07 \times 3 \mathrm{H}), 3.26(\mathrm{~s}, 0.93 \times 3 \mathrm{H}), 3.16(\mathrm{dd}, J=9.0,12.6 \mathrm{~Hz}, 0.93 \mathrm{H}), 3.07$ (d, J=10.2 Hz, $0.93 \mathrm{H}), 3.06$ (d, $J=10.8 \mathrm{~Hz}, 0.07 \mathrm{H}), 2.89$ (s, 0.07 x $3 \mathrm{H}), 2.84$ (dd, $J=5.8,11.9 \mathrm{~Hz}, 0.07 \mathrm{H}), 2.82$ (s, $0.93 \times 3 \mathrm{H}), 2.79(\mathrm{dd}, J=11.9,14.4 \mathrm{~Hz}, 0.07 \mathrm{H}), 2.47-2.38(\mathrm{~m}, 0.07 \mathrm{H}), 2.29$ (dd, $J=3.6,13.5 \mathrm{~Hz}$, $0.07 \mathrm{H}), 2.27$ (dd, $J=6.0,14.4 \mathrm{~Hz}, 0.93 \mathrm{H}$ ), 2.24 (mddd, $J=1.8,2.4,12.6 \mathrm{~Hz}, 0.093 \mathrm{H}), 2.17-2.11$ (m, $0.07 \mathrm{H}$ ), 2.09 (qdd, $J=3.0,5.4,15.0 \mathrm{~Hz}, 0.93 \mathrm{H}$ ), 2.04 (mdd, $J=7.8,15.8 \mathrm{~Hz}, 0.07 \mathrm{H}), 2.01-1.95$ $(\mathrm{m}, 0.93 \mathrm{H}), 1.90-1.86(\mathrm{~m}, 0.93 \mathrm{H}), 1.85$ (t, $J=13.0 \mathrm{~Hz}, 0.07 \mathrm{H}), 1.63-1.60(\mathrm{~m}, 0.07 \mathrm{H}), 1.60-1.55$ $(\mathrm{m}, 0.93 \mathrm{H}), 0.94(\mathrm{~d}, J=7.2 \mathrm{~Hz}, 0.93 \times 3 \mathrm{H}), 0.86(\mathrm{~s}, 0.07$ x $3 \mathrm{H}), 0.85$ (d, J=6.6 Hz, $0.93 \times 3 \mathrm{H}), 0.84$ (s, $0.93 \times 3 \mathrm{H}), 0.84(\mathrm{~d}, J=7.8 \mathrm{~Hz}, 0.07 \times 3 \mathrm{H}), 0.79(\mathrm{~d}, J=7.2 \mathrm{~Hz}, 0.07 \times 3 \mathrm{H}), 0.78(\mathrm{~s}, 0.07 \times 3 \mathrm{H})$, $0.77(\mathrm{~s}, 0.93 \times 3 \mathrm{H}) ;{ }^{13} \mathrm{C} \mathrm{NMR}\left(150 \mathrm{MHz}, \mathrm{CDCl}_{3}\right)$ for the major isomer $\delta 166.4,165.6,137.1$, 133.3, 133.2, 131.0, 130.4, 130.3, 129.3, 128.6, 127.0, 116.0, 99.9, 86.8, 79.0, 78.6, 75.4, 74.4, 73.0, 72.1, 64.7, 61.3, 47.5, 41.6, 38.2, 35.1, 34.2, 34.2, 29.0, 23.1, 18.3, 13.6, 13.1; LRMS m/z $731\left(\mathrm{M}^{+}\right)$; HRMS calcd for $\mathrm{C}_{37} \mathrm{H}_{49} \mathrm{NO}_{9} \mathrm{Se} 731.2573$, found: 731.2571 .

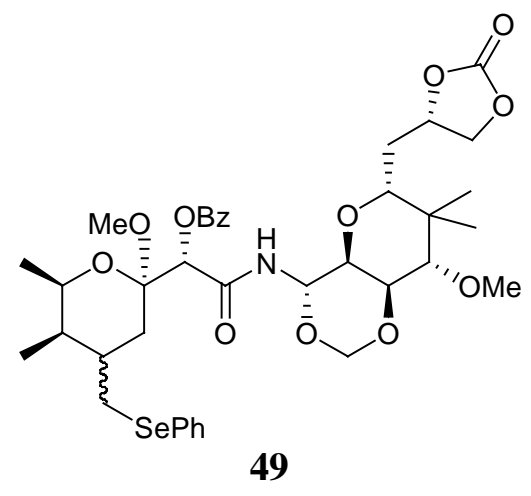

(4RS)-7-Benzoyl-17,18- $O$-carbonyldioxy-4-phenylselenylmethylmycalamide A (49). То а well stirred suspension of (DHQ) ${ }_{2} \mathrm{PYR}(3.6 \mathrm{mg}, 4.1 \mu \mathrm{mol}), \mathrm{K}_{3} \mathrm{Fe}(\mathrm{CN})_{6}(13.5 \mathrm{mg}, 41.0 \mu \mathrm{mol})$ and $\mathrm{K}_{2} \mathrm{CO}_{3}(5.7 \mathrm{mg}, 4.1 \mu \mathrm{mol})$ in ${ }^{t} \mathrm{BuOH}-\mathrm{H}_{2} \mathrm{O}(1: 1,1 \mathrm{~mL})$ was added $\mathrm{OsO}_{4}(1 \%$ in water, $5 \mu \mathrm{L}, 0.1$ $\mu \mathrm{mol})$ at $0{ }^{\circ} \mathrm{C}$. The solution was stirred for $2 \mathrm{~h}$ and poured onto alkene 48 (3.0 mg, $\left.4.1 \mu \mathrm{mol}\right)$ in a $10 \mathrm{~mL}$ flask at $-10{ }^{\circ} \mathrm{C}$. The mixture was stirred for $4 \mathrm{~h}$ at $-10{ }^{\circ} \mathrm{C}$ before saturated $\mathrm{Na}_{2} \mathrm{SO}_{3}$ solution $(2 \mathrm{~mL})$ was added. The resulting colorless solution was extracted with EtOAc $(10 \mathrm{~mL} \times 3)$. The combined organic extracts were dried $\left(\mathrm{Na}_{2} \mathrm{SO}_{4}\right)$ and concentrated to give a white solid (13 mg), which was used immediately in the next step.

To a solution of the crude diol $(13 \mathrm{mg})$ in $\mathrm{CH}_{2} \mathrm{Cl}_{2}(1 \mathrm{~mL})$ were added $\mathrm{Et}_{3} \mathrm{~N}(5.7 \mu \mathrm{L}, 41.0 \mu \mathrm{mol})$ and a solution of triphosgene $(4.9 \mathrm{mg}, 16.4 \mu \mathrm{mol})$ in $\mathrm{CH}_{2} \mathrm{Cl}_{2}(0.3 \mathrm{~mL})$. The mixture was stirred for $2 \mathrm{~h}$ and poured onto a mixture of $\mathrm{CH}_{2} \mathrm{Cl}_{2}(2 \mathrm{~mL})$ and saturated $\mathrm{NaHCO}_{3}$ solution $(2 \mathrm{~mL})$. The phases were separated and the aqueous phase was further extracted with $\mathrm{CH}_{2} \mathrm{Cl}_{2}$ (4 mL x 3). The combined organic extracts were dried $\left(\mathrm{Na}_{2} \mathrm{SO}_{4}\right)$ and concentrated to give a crude oil. Purification of the crude oil by flash chromatography (40\% EtOAc/hexane) provided the corresponding carbonates (3.1 mg, $94 \%$ for 2 steps, $\mathrm{C} 17 \alpha: \mathrm{C} 17 \beta=4.8: 1)$ as a white oil. The diastereoisomers were separated by HPLC $\left(\mathrm{SiO}_{2}, 30 \%\right.$ EtOAc/hexane) to afford carbonates 49 (1.8 mg). 
Data for 49 (C4 $\alpha . \mathrm{C} 4 \beta=11: 1$ ): IR(neat) 3352, 2959, 2930, 1799, 1732, 1699, 1271, 1124, 1107 , $1092,1069,1036,712 \mathrm{~cm}^{-1} ;{ }^{1} \mathrm{H}$ NMR $\left(400 \mathrm{MHz}, \mathrm{C}_{6} \mathrm{D}_{6}\right.$ referenced to $\left.7.15 \mathrm{ppm}\right) \delta 8.30(\mathrm{dd}, J=2.8$, $7.6 \mathrm{~Hz}, 2 \mathrm{H}), 7.54(\mathrm{~d}, J=7.1 \mathrm{~Hz}, 0.92 \times 2 \mathrm{H}), 7.53(\mathrm{~d}, J=8.3 \mathrm{~Hz}, 0.08 \times 2 \mathrm{H}), 7.31(\mathrm{~d}, J=11.0 \mathrm{H}, 1 \mathrm{H})$, 7.12-6.95 (m, $6 \mathrm{H}), 5.79$ (s, $0.08 \mathrm{H}), 5.76(\mathrm{~s}, 0.92 \mathrm{H}), 4.50$ (t, J=9.5 Hz, $1 \mathrm{H}), 4.81-4.74$ (m, $0.08 \mathrm{x}$ $2 \mathrm{H}), 4.76-4.69(\mathrm{~m}, 0.92 \times 2 \mathrm{H}), 4.61(\mathrm{t}, J=8.5 \mathrm{~Hz}, 0.08 \mathrm{H}), 4.54$ (t, J=8.3 Hz, $0.92 \mathrm{H}), 4.52$ (d, $J=6.8 \mathrm{~Hz}, 0.92 \mathrm{H}), 4.51(\mathrm{~d}, J=6.9 \mathrm{~Hz}, 0.08 \mathrm{H}), 4.40$ (d, J=6.8 Hz, $0.92 \mathrm{H}), 4.39$ (d, J=6.9 Hz, 0.08 H), $4.19(\mathrm{dd}, J=7.1,10.7 \mathrm{~Hz}, 0.08 \mathrm{H}), 4.18(\mathrm{dd}, J=6.8,10.5 \mathrm{~Hz}, 0.08 \mathrm{H}), 3.68(\mathrm{dd}, J=7.1,10.0 \mathrm{~Hz}$, $0.08 \mathrm{H}), 3.71(\mathrm{dq}, J=2.4,6.2 \mathrm{~Hz}, 0.92 \mathrm{H}), 3.68(\mathrm{dd}, J=7.1,10.0 \mathrm{~Hz}, 0.08 \mathrm{H}), 3.54(\mathrm{dd}, J=8.0,8.0$ $\mathrm{Hz}, 0.08 \mathrm{H}), 3.54(\mathrm{dd}, J=6.2,8.3 \mathrm{~Hz}, 0.08 \mathrm{H}), 3.53(\mathrm{dd}, J=6.1,8.5 \mathrm{~Hz}, 0.92 \mathrm{H}), 3.24(\mathrm{~s}, 3 \mathrm{H}), 3.23$ (dd, $J=6.6,12.4 \mathrm{~Hz}, 0.92 \mathrm{H}), 3.14$ (dd, $J=9.0,12.4 \mathrm{~Hz}, 0.92 \mathrm{H}), 3.09$ (d, $J=10.5 \mathrm{~Hz}, 0.92 \mathrm{H}), 3.06$ (d, $J=10.1 \mathrm{~Hz}, 0.08 \mathrm{H}), 2.83(\mathrm{dd}, J=6.5,11.9 \mathrm{~Hz}, 0.08 \mathrm{H}), 2.79-2.77(\mathrm{~m}, 0.08 \mathrm{H}), 2.82$ (s, 0.08 x 3 H), 2.78 (s, $0.92 \times 3 \mathrm{H}), 2.38-2.30(\mathrm{~m}, 0.08 \mathrm{H}), 2.24$ (dd, $J=11.3,14.8 \mathrm{~Hz}, 0.08 \mathrm{H}), 2.25$ (dd, $J=2.8$, $11.7 \mathrm{~Hz}, 0.92 \mathrm{H}), 2.19$ (dd, $J=3.2,13.0 \mathrm{~Hz}, 0.08 \mathrm{H}), 2.04$ (dd, $J=6.1,14.1 \mathrm{~Hz}, 0.92 \mathrm{H}), 1.96-1.88$ $(\mathrm{m}, 1 \mathrm{H}), 1.65-1.54(\mathrm{~m}, 2 \mathrm{H}), 1.16(\mathrm{dd}, J=10.0,13.9 \mathrm{~Hz}, 1 \mathrm{H}), 0.88(\mathrm{~d}, J=7.1 \mathrm{~Hz}, 0.92 \times 3 \mathrm{H}), 0.83$ $(\mathrm{d}, J=6.3 \mathrm{~Hz}, 0.92 \times 3 \mathrm{H}), 0.82(\mathrm{~d}, J=6.9 \mathrm{~Hz}, 0.08 \times 3 \mathrm{H}), 0.72(\mathrm{~d}, J=7.1 \mathrm{~Hz}, 0.08 \times 3 \mathrm{H}), 0.66$ (s, $3 \mathrm{H}), 0.82(\mathrm{~d}, J=6.9 \mathrm{~Hz}, 0.08 \times 3 \mathrm{H}), 0.72(\mathrm{~d}, J=7.1 \mathrm{~Hz}, 0.08 \times 3 \mathrm{H}), 0.66(\mathrm{~s}, 3 \mathrm{H}), 0.54(\mathrm{~s}, 3 \mathrm{H}) ;{ }^{13} \mathrm{C}$ NMR (150 MHz, $\mathrm{C}_{6} \mathrm{D}_{6}$ referenced to $128 \mathrm{ppm}$ ) for the major isomer $\delta 166.8,165.5,133.8,133.3$, 132.6, 130.8, 130.1, 129.8, 129.5, 129.0, 128.4, 127.3, 127.2, 99.9, 86.8, 78.3, 74.9, 74.8, 74.7, 72.7, 72.6, 69.9, 64.9, 62.8, 61.4, 47.7, 41.3, 38.5, 34.8, 34.4, 33.9, 30.6, 28.7, 22.8, 18.3, 13.0; LRMS $m / z 791\left(\mathrm{M}^{+}\right)$; HRMS calcd for $\mathrm{C}_{38} \mathrm{H}_{49} \mathrm{NO}_{12} \mathrm{Se}$ 791.2420, found: 791.2412. 